\title{
RATE OF CONVERGENCE OF GENERALIZED HERMITE-PADÉ APPROXIMANTS OF NIKISHIN SYSTEMS
}

\author{
U. FIDALGO PRIETO AND G. LÓPEZ LAGOMASINO
}

\begin{abstract}
We study the rate of convergence of interpolating simultaneous rational approximations with partially prescribed poles to so called Nikishin systems of functions. To this end, a vector equilibrium problem in the presence of a vector external field is solved which is used to describe the asymptotic behavior of the corresponding second type functions which appear.
\end{abstract}

\section{Generalized Hermite-Padé approximants}

Let $\Delta_{1}$ be a bounded interval of the real line $\mathbb{R}$. By $\mathcal{M}\left(\Delta_{1}\right)$ we denote the set of all Borel measures with constant sign (positive or negative) whose $\operatorname{support} \operatorname{supp}(\cdot)$ is contained in $\Delta_{1}$ and contains infinitely many points. Let $s=\left(s_{1}, \cdots s_{m}\right)$ be a vector of measures belonging to $\mathcal{M}\left(\Delta_{1}\right)$. The Markov function corresponding to the measure $s_{i} \in \mathcal{M}\left(\Delta_{1}\right)$ is given by

$$
\widehat{s}_{i}(z)=\int_{\Delta_{1}} \frac{d s_{i}(x)}{z-x} .
$$

Certainly, $\widehat{s}_{i}$ is a holomorphic function in $\overline{\mathbb{C}} \backslash \Delta_{1}$.

We restrict our attention to a special class of vector measures introduced by E. M. Nikishin in [11] and adopt the notation introduced in [8]. Let $\sigma_{1}$ and $\sigma_{2}$ be two measures supported on $\mathbb{R}$ with constant sign and let $\Delta_{1}, \Delta_{2}$ denote the convex hull of $\operatorname{supp}\left(\sigma_{1}\right)$ and $\operatorname{supp}\left(\sigma_{2}\right)$ respectively; that is, $\Delta_{i}=\operatorname{Co}\left(\operatorname{supp}\left(\sigma_{i}\right)\right)$. Suppose that $\Delta_{1} \cap \Delta_{2}=\emptyset$. Set

$$
d\left\langle\sigma_{1}, \sigma_{2}\right\rangle(x)=\int \frac{d \sigma_{2}(t)}{x-t} d \sigma_{1}(x)=\widehat{\sigma}_{2}(x) d \sigma_{1}(x) .
$$

When it is convenient we use the differential notation of a measure. Then $\left\langle\sigma_{1}, \sigma_{2}\right\rangle$ is a measure with constant sign and supported on $\operatorname{supp}\left(\sigma_{1}\right) \subset \Delta_{1}$.

Definition 1. Given a system of closed bounded intervals $\Delta_{1}, \ldots, \Delta_{m}$ satisfying $\Delta_{j-1} \cap \Delta_{j}=$ $\emptyset, j=2, \ldots, m$, and finite Borel measures $\sigma_{1}, \ldots, \sigma_{m}$ with constant sign and $\operatorname{Co}\left(\operatorname{supp}\left(\sigma_{j}\right)\right)=\Delta_{j}$, we define inductively

$$
s_{j}=\left\langle\sigma_{1}, \sigma_{2}, \cdots, \sigma_{j}\right\rangle=\left\langle\sigma_{1},\left\langle\sigma_{2}, \cdots, \sigma_{j}\right\rangle\right\rangle, \quad j=2, \cdots m .
$$

We say that $S=\left(s_{1}, \cdots, s_{m}\right)=\mathcal{N}\left(\sigma_{1}, \cdots, \sigma_{m}\right)$ with $s_{1}=\sigma_{1}$ is the Nikishin system generated by the measures $\left(\sigma_{1}, \ldots, \sigma_{m}\right)$.

Given a Nikishin system $S=\left(s_{1}, \ldots, s_{m}\right)=\mathcal{N}\left(\sigma_{1}, \cdots, \sigma_{m}\right)$ denote by $\widehat{S}=\left(\widehat{s}_{1}, \ldots \widehat{s}_{m}\right)$ the vector whose components are the Markov functions corresponding to each one of the measures $s_{i}$, $i=1, \ldots, m$. The functions $\widehat{s}_{i}, i=1, \ldots, m$, are holomorphic in $D=\overline{\mathbb{C}} \backslash \Delta_{1}$. Fix a multi-index $n=\left(n_{1}, \cdots, n_{m}\right) \in \mathbb{Z}_{+}^{m}$, where $\mathbb{Z}_{+}=\{0,1,2, \ldots\}$, and an even integer $\kappa$. Set $|n|=\sum_{i=1}^{m} n_{i}$. Let $\alpha$ and $\beta$ be two monic polynomials with real coefficients such that $\operatorname{deg} \beta=\kappa$ and $\operatorname{deg} \alpha \leq$ $|n|+\kappa+\min \left\{n_{i}\right\}$. The zeros of $\alpha$ belong to $D$ and the zeros of $\beta$ have even multiplicity and lie on $\Delta_{1}$. There exists a vector of rational functions $R=\left(P_{1} / \beta Q, \cdots, P_{m} / \beta Q\right)$, such that

i) $\operatorname{deg} Q \leq|n|, \quad Q \not \equiv 0, \quad \operatorname{deg} P_{i} \leq|n|+\kappa-1, \quad i=1, \cdots, m$,

ii) $\left[\frac{\beta Q \widehat{s}_{i}-P_{i}}{\alpha}\right](z)=O\left(\frac{1}{z^{n_{i}+1}}\right) \in \mathcal{H}(D), \quad i=1, \cdots m$.

1991 Mathematics Subject Classification. Primary 42C05.

The work of both authors was supported by Ministerio de Ciencia y Tecnología under grant BFM 2003-06335C03-02. The second author was also partially supported by NATO PST.CLG.979738 and INTAS 03-51-6637. 
Proving the existence of $R$ reduces to solving a system of $(m+1)|n|+m \kappa$ homogeneous linear equations on $(m+1)|n|+m \kappa+1$ unknowns, and this always has a non trivial solution. We call the vector rational function $R$ a generalized Hermite-Padé approximant (GHPA) of $\widehat{S}$ relative to $(n, \alpha, \beta)$. When $\alpha \equiv \beta \equiv 1$ generalized Hermite-Padé reduce to classical Hermite-Padé.

It is well known that the denominator $Q$ of classical Hermite-Padé approximants share orthogonality relations with each measure in the system $S$. An analogous result takes place for GHPA. In fact, from ii) we have that

$$
z^{\nu}\left[\frac{\beta Q \widehat{s}_{i}-P_{i}}{\alpha}\right](z)=O\left(\frac{1}{z^{2}}\right) \in \mathcal{H}(D), \quad \nu=0, \ldots, n_{i}-1, \quad i=1, \ldots m .
$$

Let $\Gamma$ be a closed curve with winding number 1 for all its interior points such that all the zeros of $\alpha$ lie exterior to $\Gamma$ and $\Delta_{1}$ is surrounded by $\Gamma$. Integrating (2) along $\Gamma$ and using Cauchy's Theorem, we obtain

$$
0=\int_{\Gamma} z^{\nu}\left[\frac{\beta Q \widehat{s}_{i}-P_{i}}{\alpha}\right](z) d z=\int_{\Gamma} z^{\nu} \frac{\beta Q \widehat{s}_{i}}{\alpha}(z) d z, \quad \nu=0, \ldots, n_{i}-1, \quad i=1, \ldots m .
$$

Substituting (1) in (3), using Fubini's Theorem, and Cauchy's Integral Formula, it follows that

$$
\int_{\Delta_{1}} x^{\nu} Q(x) \frac{\beta(x) d s_{i}(x)}{\alpha(x)}=\int_{\Delta_{1}} x^{\nu} Q(x) d \widetilde{s}_{i}(x)=0, \quad \nu=0, \ldots, n_{i}-1, \quad i=1, \ldots, m,
$$

where

$$
d \widetilde{s}_{i}=\frac{\beta}{\alpha} d s_{i}, \quad i=1, \ldots, m .
$$

Notice that $\widetilde{s}_{i}=\left(\widetilde{s}_{1}, \ldots, \widetilde{s}_{m}\right)=\mathcal{N}\left(\vartheta_{1}, \sigma_{2}, \ldots, \sigma_{m}\right)$, where

$$
d \vartheta_{1}=\frac{\beta}{\alpha} d \sigma_{1} .
$$

In Section 6 we study the rate of convergence of sequences $\left\{R_{n}\right\}, n \in \Lambda$, of GHPA (see Theorem 7 and Corollary 2) where $\Lambda \subset \mathbb{Z}_{+}^{m}$ is a sequence of multi-indices. To this end, it is important to study the logarithmic asymptotic behavior of the corresponding sequence $\left\{Q_{n}\right\}, n \in \Lambda$, of common denominators which is done in Section 5 (see Theorem 5). The instruments used are potential theoretic arguments developed in Section 4 (see Theorem 4) relative to the existence of the solution of a vector equilibrium problem in the presence of a vector external field and some orthogonality relations satisfied by second type functions associated with the Nikishin system of functions described in Section 3 (see Theorems 2 and 3). In all this the normality of the multiindices considered play a crucial role. The class of multi-indices from which the sequence $\Lambda$ is extracted is introduced in Section 2.

\section{Normality of indices of GHPA.}

In general, the uniqueness of $Q$ is not known to occur. When for a multi-index $n, Q$ is uniquely determined, we say that $n$ is weakly normal. A multi-index $n$ is said to be normal if the conditions i)-ii) imply that $\operatorname{deg} Q=|n|$. If additionally the $|n|$ zeros of $Q$ are simple and lie in the interior of the interval $\Delta_{1}$ then $n$ is said to be strongly normal. (In reference to the interior of intervals of the real line we consider the usual Euclidean topology of $\mathbb{R}$.) Obviously, strong normality implies normality and this in turn implies weak normality. Systems $S$ for which all multi-indices $n$ are weakly normal, normal, or strongly normal are called weakly perfect, perfect, or strongly perfect, respectively.

In [11], E. M. Nikishin defines AT systems. This concept is very appropriate in proving strong normality

DEFINITION 2. Let $g_{i}, i=1, \ldots, m$, be continuous functions with constant sign on an interval $F$. We say that $\left(g_{1}, \ldots, g_{m}\right)$ defines an AT system for the multi-index $n=\left(n_{1}, \ldots, n_{m}\right)$ on $F$ if for any polynomials $h_{1}, \cdots, h_{m}$ such that $\operatorname{deg} h_{i} \leq n_{i}-1, i=1, \cdots, m$, not all simultaneously identically equal to zero, the function

$$
H_{n}(x)=H\left(h_{1}, \ldots, h_{m} ; x\right)=h_{1}(x) g_{1}(x)+\cdots+h_{m}(x) g_{m}(x) .
$$

has at most $|n|-1$ zeros on $F\left(\operatorname{deg} h_{i} \leq-1\right.$ means that $\left.h_{i} \equiv 0\right)$. The system $\left(g_{1}, \ldots, g_{m}\right)$ is an AT system on $F$, if it defines an AT system on that interval for all multi-indices $n \in \mathbb{Z}_{+}^{m}$. 
We denote

- $\mathbb{Z}_{+}^{m}(*)=\left\{n \in \mathbb{Z}_{+}^{m}\right.$ : there do not exist $i<j<k$ such that $\left.n_{i}<n_{j}<n_{k}\right\}$.

- For each $i=1, \ldots, m$, we introduce the measures

$$
s_{i, j}=\left\langle\sigma_{i}, \ldots, \sigma_{j}\right\rangle, \quad j=i, \ldots, m
$$

Denoting $\widehat{s}_{2,1} \equiv 1$, according to the orthogonality conditions (4), we have

$$
\int_{\Delta_{1}} Q(x)\left(h_{1} \widehat{s}_{2,1}+\cdots+h_{m} \widehat{s}_{2, m}\right)(x) d \vartheta_{1}(x)=0,
$$

where $\operatorname{deg} h_{i} \leq n_{i}-1, i=1, \ldots, m$. Assume that for $n=\left(n_{1}, \ldots, n_{m}\right)$ the system of functions $\left(\widehat{s}_{2,1}, \widehat{s}_{2,2}, \ldots, \widehat{s}_{2, m}\right)$ defines an AT system on $\Delta_{1}$. Using (5) it follows that $\operatorname{deg} Q_{n}=|n|$ and $|n|$ is strongly normal. Using this argument, in [4] and [5] a large class of strongly normal multi-indices is proved to exist. For convenience of the reader, we state the corresponding result.

Theorem 1. Assume that either $1 \leq m \leq 3$ and $n \in \mathbb{Z}_{+}^{m}$, or $m \geq 4$ and $n \in \mathbb{Z}_{+}^{m}(*)$, then $\left(\widehat{s}_{2,1}, \widehat{s}_{2,2}, \ldots, \widehat{s}_{2, m}\right)$ defines an AT system with respect to $n$ on any interval disjoint from $\Delta_{2}$ and, consequently, $n$ is strongly normal.

Notice that this means that under the assumptions of Theorem 1 a system of functions formed by 1 and a Nikishin system of functions determines an AT system on any closed interval disjoint from the support of the measures integrating the Nikishin system of measures. In this form this result will be used below in the proof of Theorem 2 (see the last statement of the proof).

\section{FunCtions OF SECOND TYPE AND ORTHOGONALITY}

Let $n \in \mathbb{Z}_{+}^{m}(*)$ and $\Sigma=\left(\sigma_{1}, \ldots, \sigma_{m}\right)$ be given such that $S=\mathcal{N}(\Sigma)$ forms a Nikishin system of measures. Take $\Psi_{0}=Q \beta / \alpha$ where $Q$ is the common denominator of the GHPA of $\widehat{S}$ relative to $(n, \alpha, \beta)$. Set $n=n^{0}$ and $\Sigma=\Sigma^{0}$. Inductively, we will construct multi-indices $n^{j} \in \mathbb{Z}_{+}^{m-j}(*)$, vectors of measures $\Sigma^{j}=\left(\sigma_{j+1}^{j}, \ldots, \sigma_{m}^{j}\right)$, and second type functions $\Psi_{j}, j=1, \ldots, m-1$, as described below.

Assume that $n^{j}=\left(n_{j+1}^{j}, \ldots, n_{m}^{j}\right), \Sigma^{j}=\left(\sigma_{j+1}^{j}, \ldots, \sigma_{m}^{j}\right)$, and $\Psi_{j}$ have been defined, where $0 \leq j \leq m-2$. Then,

$$
n^{j+1}=\left(n_{j+2}^{j+1}, \ldots, n_{m}^{j+1}\right) \in \mathbb{Z}_{+}^{m-j-1}
$$

is the multi-index obtained extracting from $n^{j}$ the first component $n_{r_{j}}^{j}$ satisfying

$$
n_{r_{j}}^{j}=\max \left\{n_{k}^{j}: j+1 \leq k \leq m\right\}
$$

Therefore,

$$
n_{j+1}^{j}=n_{j+2}^{j+1}, \ldots, n_{r_{j}-1}^{j}=n_{r_{j}}^{j+1}, n_{r_{j}+1}^{j}=n_{r_{j}+1}^{j+1}, \ldots, n_{m}^{j}=n_{m}^{j+1}
$$

and when $r_{j} \geq j+2$

$$
n_{r_{j}}^{j}>\max \left\{n_{j+1}^{j}, \ldots, n_{r_{j}-1}^{j}\right\}
$$

Notice that if $n \in \mathbb{Z}_{+}^{m}(*)$ then

$$
n_{j+2}^{j+1} \geq \cdots \geq n_{r_{j}}^{j+1}
$$

since otherwise $n^{j} \notin \mathbb{Z}_{+}^{m-j}(*)$. Set

$$
\Psi_{j+1}(z)=\int_{\Delta_{j+1}} \frac{\Psi_{j}(x)}{z-x} d s_{r_{j}}^{j}(x),
$$

where $s_{r_{j}}^{j}=\left\langle\sigma_{j+1}^{j}, \ldots, \sigma_{r_{j}}^{j}\right\rangle$ is the component of $\mathcal{N}\left(\Sigma^{j}\right)=\left(s_{j+1}^{j}, \ldots, s_{m}^{j}\right)=S^{j}$ corresponding to the subindex $r_{j}$.

Before defining $\Sigma^{j+1}$, we need some more notation. For the system $\Sigma^{j}=\left(\sigma_{j+1}^{j}, \ldots, \sigma_{m}^{j}\right)$ we define

$$
s_{k, i}^{j}=\left\langle\sigma_{k}^{j}, \ldots, \sigma_{i}^{j}\right\rangle, \quad j+1 \leq k \leq i \leq m
$$


In particular, the measures in $\mathcal{N}\left(\Sigma^{j}\right)$ are $s_{i}^{j}=s_{j+1, i}^{j}, j+1 \leq i \leq m$. Given any measure $s_{k, i}^{j}$ there exists a first degree polynomial $\ell_{k, i}^{j}$ and a measure with constant sign $\tau_{k, i}^{j}$ such that

$$
\frac{1}{\widehat{s}_{k, i}^{j}(z)}=\ell_{k, i}^{j}(z)+\widehat{\tau}_{k, i}^{j}(z) \text {. }
$$

For a proof see the Appendix in [9].

Suppose that $r_{j}=j+1$, then

$$
\Sigma^{j+1}=\left(\sigma_{j+2}^{j}, \ldots, \sigma_{m}^{j}\right)=\left(\sigma_{j+2}^{j+1}, \ldots, \sigma_{m}^{j+1}\right)
$$

is the vector of $m-j-1$ measures which is obtained deleting from $\Sigma^{j}$ its first component. If $j+2 \leq r_{j} \leq m$, then

$$
\Sigma^{j+1}=\left(\tau_{j+2, r_{j}}^{j}, \widehat{s}_{j+2, r_{j}}^{j} d \tau_{j+3, r_{j}}^{j}, \ldots, \widehat{s}_{r_{j}-1, r_{j}}^{j} d \tau_{r_{j}, r_{j}}^{j}, \widehat{s}_{r_{j}, r_{j}}^{j} d \sigma_{r_{j}+1}^{j}, \sigma_{r_{j}+2}^{j}, \ldots, \sigma_{m}^{j}\right) .
$$

The two results that follow are analogous to Propositions 1-3 in [8] but their proof is technically more complicated.

Theorem 2. Assume that $n \in \mathbb{Z}_{+}^{m}(*)$. For each $j=0, \ldots, m-1$, we have that $\Psi_{j}$ satisfies the orthogonality relations

$$
\int_{\Delta_{j+1}} x^{\nu} \Psi_{j}(x) d s_{i}^{j}(x)=0, \quad \nu=0, \ldots, n_{i}^{j}-1, \quad i=j+1, \ldots, m .
$$

Consequently, we can write,

$$
\frac{\Psi_{j}(x)}{q_{j+1}(x)}=\psi_{j}(x) \in \mathcal{H}\left(\overline{\mathbb{C}} \backslash \Delta_{j}\right), \quad j=1, \ldots m-1,
$$

where $q_{j+1}$ is a monic polynomial whose zeros are simple, lie in the interior of $\Delta_{j+1}$, $\operatorname{deg} q_{j+1} \geq\left|n^{j}\right|$ and $\psi_{j}$ preserves the same sign on $\Delta_{j+1}$.

Proof. We shall prove (8) by induction on $j$. For $j=0$ the relations (8) coincide with (4). Thus, for $j=0$ the statement holds, and if $m=1$ we have concluded the proof. Let us assume that these relations are verified for some fixed $j, 0 \leq j \leq m-2, m \geq 2$, and let us prove that

$$
\int_{\Delta_{j+2}} x^{\nu} \Psi_{j+1}(x) d s_{i}^{j+1}(x)=0, \quad \nu=0, \ldots, n_{i}^{j+1}-1, \quad i=j+2, \ldots, m .
$$

Take $i \in\{j+2, \ldots, m\}$ and $0 \leq \nu \leq n_{i}^{j+1}-1$. Substituting $\Psi_{j+1}$ defined by (7) into the left hand of (10), we have

$$
\begin{gathered}
\int_{\Delta_{j+2}} x^{\nu} \Psi_{j+1}(x) d s_{i}^{j+1}(x)=\int_{\Delta_{j+2}} x^{\nu} \int_{\Delta_{j+1}} \frac{\Psi_{j}(t)}{x-t} d s_{r_{j}}^{j}(t) d s_{i}^{j+1}(x)= \\
\int_{\Delta_{j+1}} \Psi_{j}(t) \int_{\Delta_{j+2}} \frac{x^{\nu}-t^{\nu}+t^{\nu}}{x-t} d s_{i}^{j+1}(x) d s_{r_{j}}^{j}(t)= \\
\int_{\Delta_{j+1}} p_{\nu}(t) \Psi_{j}(t) d s_{r_{j}}^{j}(t)-\int_{\Delta_{j+1}} t^{\nu} \Psi_{j}(t) \widehat{s}_{i}^{j+1}(t) d s_{r_{j}}^{j}(t),
\end{gathered}
$$

where $\operatorname{deg} p_{\nu} \leq n_{i}^{j+1}-2$. Taking into consideration that $n_{i}^{j+1}-2 \leq n_{r_{j}}^{j}-1$ (see the definition (6) of $r_{j}$ ) and the induction hypothesis, the first of the last two integrals equals zero. Therefore,

$$
\int_{\Delta_{j+2}} x^{\nu} \Psi_{j+1}(x) d s_{i}^{j+1}(x)=-\int_{\Delta_{j+1}} t^{\nu} \Psi_{j}(t) \widehat{s}_{i}^{j+1}(t) d s_{r_{j}}^{j}(t) .
$$

If $r_{j}=j+1$ then $\Sigma^{j+1}=\left(\sigma_{j+2}^{j+1}, \ldots, \sigma_{m}^{j+1}\right)=\left(\sigma_{j+2}^{j}, \ldots, \sigma_{m}^{j}\right)$ and $n^{j+1}=\left(n_{j+2}^{j+1}, \ldots, n_{m}^{j+1}\right)=$ $\left(n_{j+2}^{j}, \ldots, n_{m}^{j}\right)$. Then,

$$
\widehat{s}_{i}^{j+1}(t) d s_{r_{j}}^{j}(t)=\left\langle\sigma_{j+1}^{j},\left\langle\sigma_{j+2}^{j}, \ldots, \sigma_{i}^{j}\right\rangle\right\rangle=s_{i}^{j} .
$$

Since in this case $n_{i}^{j+1}=n_{i}^{j}$, the right hand of (11) equals zero by the induction hypothesis and we have proved what we needed. 
Let us suppose that $r_{j} \geq j+2$. In order to consider this situation we need some less obvious relations between the measures in $S^{j}$ and $S^{j+1}$. Their proof may be found in Theorem 3.1.3 of [3]. In order to avoid confusion, we state these relations with the notation introduced above. We have

$$
\begin{gathered}
\frac{\widehat{s}_{j+2, i-1}^{j}}{\widehat{s}_{j+2, r_{j}}^{j}}=a_{i, j}+\widehat{s}_{i}^{j+1}+c_{i, j} \widehat{s}_{i-1}^{j+1}, \quad j+3 \leq i \leq r_{j}, \\
\frac{1}{\widehat{s}_{j+2, r_{j}}^{j}}=\ell_{j}+\widehat{s}_{j+2}^{j+1},
\end{gathered}
$$

and

$$
\frac{\widehat{s}_{j+2, i}^{j}}{\widehat{s}_{j+2, r_{j}}^{j}}=a_{i, j}+\widehat{s}_{i}^{j+1}, \quad r_{j}+1 \leq i \leq m,
$$

where $a_{i, j}, c_{i, j}$ are constants and $\ell_{j}$ is a first degree polynomial. Relation (13) was already mentioned above in a more general context. We repeat it here to unify the notation.

First let us consider that $r_{j}+1 \leq i \leq m$. If $r_{j}=m$ this is an empty set and we have nothing to prove. Assume that $j+2 \leq r_{j} \leq m-1$. Substituting (14) into the right hand of (11), we obtain

$$
\begin{gathered}
\int_{\Delta_{j+2}} x^{\nu} \Psi_{j+1}(x) d s_{i}^{j+1}(x)=-\int_{\Delta_{j+1}} t^{\nu} \Psi_{j}(t)\left(\frac{\widehat{s}_{j+2, i}^{j}(t)}{\hat{s}_{j+2, r_{j}}^{j}(t)}-a_{i, j}\right) d s_{r_{j}}^{j}(t)= \\
a_{i, j} \int_{\Delta_{j+1}} t^{\nu} \Psi_{j}(t) d s_{r_{j}}^{j}(t)-\int_{\Delta_{j+1}} t^{\nu} \Psi_{j}(t) \widehat{s}_{j+2, i}^{j}(t) d \sigma_{j+1}^{j}(t) .
\end{gathered}
$$

Since $\widehat{s}_{j+2, i}^{j}(t) d \sigma_{j+1}^{j}(t)=d s_{i}^{j}(t)$ and $0 \leq \nu \leq n_{i}^{j+1}-1=n_{i}^{j}-1 \leq n_{r_{j}}^{j}-1$, by the induction hypothesis the last two integrals vanish and we obtain (10) for these indices $i$.

Now, let $j+2 \leq i \leq r_{j}$. Using (12) several times until $i-1$ reduces to $j+2($ when $i \geq j+3$ ) and once (13), we obtain

$$
\begin{gathered}
\hat{s}_{i}^{j+1}=\frac{\widehat{s}_{j+2, i-1}^{j}}{\widehat{s}_{j+2, r_{j}}^{j}}-a_{i, j}-c_{i, j} \widehat{s}_{i-1}^{j+1}= \\
\frac{\widehat{s}_{j+2, i-1}^{j}}{\widehat{s}_{j+2, r_{j}}^{j}}-a_{i, j}-c_{i, j}\left(\frac{\widehat{s}_{j+2, i-2}^{j}}{\widehat{s}_{j+2, r_{j}}^{j}}-a_{i-1, j}-c_{i-1, j} \widehat{s}_{i-2}^{j+1}\right)=\cdots=\mathcal{L}_{i, j}+\frac{1}{\widehat{s}_{j+2, r_{j}}^{j}} \sum_{k=j+1}^{i-1} c_{k} \widehat{s}_{j+2, k}^{j},
\end{gathered}
$$

where $\mathcal{L}_{i, j}$ denotes a first degree polynomial, $\widehat{s}_{j+2, j+1}^{j} \equiv 1$, and $c_{k}, k=j+1, \ldots, i-1$, are constants and $c_{i-1}=1$. Notice that

$$
\frac{\widehat{s}_{j+2, k}^{j} d s_{r_{j}}^{j}}{\widehat{s}_{j+2, r_{j}}^{j}}=\widehat{s}_{j+2, k}^{j} d \sigma_{j+1}^{j}=d s_{k}^{j}, \quad k=j+1, \ldots, i-1 .
$$

Substituting (15) into the right hand of (11) and using (16), we have

$$
\int_{\Delta_{j+2}} x^{\nu} \Psi_{j+1}(x) d s_{i}^{j+1}(x)=-\int_{\Delta_{j+1}} t^{\nu} \mathcal{L}_{i, j}(t) \Psi_{j}(t) d s_{r_{j}}^{j}(t)-\sum_{k=j+1}^{i-1} \int_{\Delta_{j+1}} t^{\nu} \Psi_{j}(t) d s_{k}^{j}(t) .
$$

Since $\nu+1 \leq n_{i}^{j+1}=n_{i-1}^{j} \leq n_{i-1}^{j+1}=n_{i-2}^{j} \leq \cdots \leq n_{j+2}^{j+1}=n_{j+1}^{j}<n_{r_{j}}^{j}$ all the integrals in the right hand of this equality vanish and we conclude the proof of (10). Therefore, the induction is complete and (8) takes place.

According to (8)

$$
\int_{\Delta_{j+1}} \Psi_{j}(x) \sum_{i=j+1}^{m} h_{i}(x) \widehat{s}_{j+2, i}^{j}(x) d \sigma_{j+1}^{j}(x)=0
$$

where $\widehat{s}_{j+2, j+1}^{j}(x) \equiv 1$ and $h_{i}, i=j+1, \ldots, m$, denote polynomials such that $\operatorname{deg} h_{i} \leq n_{i}^{j}-1$. Since $n^{j} \in \mathbb{Z}_{+}^{m-j}(*)$, by Theorem 1 the system of functions $\left(\widehat{s}_{j+2, j+1}^{j}, \widehat{s}_{j+2, j+2}^{j}, \ldots, \widehat{s}_{j+2, m}^{j}\right)$ forms an AT system on $\Delta_{j+1}$ with respect to $n^{j}$ and, consequently, from (17) it follows that $\Psi_{j}$ must have in the 
interior of $\Delta_{j+1}$ at least $\left|n^{j}\right|$ sign changes. Since $\Psi_{j}$ is not identically equal to zero, it may have only a finite number of sign changes inside $\Delta_{j+1}$. Let $q_{j+1}$ be the monic polynomial whose zeros are the distinct points inside $\Delta_{j+1}$ where $\Psi_{j}$ changes sign inside $\Delta_{j+1}$. We immediately obtain (9), that $\operatorname{deg} q_{j+1} \geq\left|n^{j}\right|$, and the sign preserving property of $\psi_{j}$.

To complete the notation, set

$$
\Psi_{m}(z)=\int_{\Delta_{m}} \frac{\Psi_{m-1}(x)}{z-x} d s_{m}^{m-1}(x) .
$$

Notice that according to (6), $r_{m-1}=m$. From (8) we have that

$$
\int_{\Delta_{j+1}} \frac{h(z)-h(x)}{z-x} \Psi_{j}(x) d s_{r_{j}}^{j}(x)=0,
$$

where $h$ is an arbitrary polynomial such that $\operatorname{deg} h \leq n_{r_{j}}^{j}, j=0, \ldots, m-1$. Using (7) and the definition of $\Psi_{m}$ given above, this last relation may be rewritten as

$$
\Psi_{j+1}(z)=\frac{1}{h(z)} \int_{\Delta_{j+1}} \Psi_{j}(x) \frac{h(x) d s_{r_{j}}^{j}(x)}{z-x}=O\left(\frac{1}{z^{n_{r_{j}}^{j}+1}}\right) \in \mathcal{H}\left(\overline{\mathbb{C}} \backslash \Delta_{j+1}\right), \quad j=0, \ldots m-1 .
$$

TheOREM 3. Assume that $n \in \mathbb{Z}_{+}^{m}(*)$. For each $j=0, \ldots, m-1$

$$
\int_{\Delta_{j+1}} x^{\nu} \Psi_{j}(x) \frac{d s_{r_{j}}^{j}(x)}{q_{j+2}(x)}=0, \quad \nu=0, \ldots\left|n^{j}\right|-1 .
$$

where $q_{j+2}$, is given in $(9),\left(q_{m+1} \equiv 1\right)$. Consequently, $\operatorname{deg} q_{j+1}=\left|n^{j}\right|, j=0, \ldots, m-1,\left(q_{1}=Q\right)$.

Proof. From (8) and (18), it follows that

$$
\frac{\Psi_{j+1}}{q_{j+2}}=O\left(\frac{1}{z^{n_{r_{j}}^{j}+\operatorname{deg} q_{j+2}+1}}\right) \in \mathcal{H}\left(\overline{\mathbb{C}} \backslash \Delta_{j+1}\right), \quad j=0, \ldots m-1
$$

Therefore,

$$
z^{\nu} \frac{\Psi_{j+1}(z)}{q_{j+2}(z)}=O\left(\frac{1}{z^{2}}\right) \in \mathcal{H}\left(\overline{\mathbb{C}} \backslash \Delta_{j+1}\right), \quad \nu=0, \ldots, n_{r_{j}}^{j}+\operatorname{deg} q_{j+2}-1, \quad j=0, \ldots m-1 .
$$

Integrating along a simple closed curve $\Gamma$ with winding number one for all its interior points which surrounds $\Delta_{j+1}$ such that $\Delta_{j+2}$ lies in the exterior of $\Gamma$, using Cauchy's Theorem, the definition of $\Psi_{j+1}$, Fubini's Theorem, and Cauchy's Integral formula, we find that

$$
\begin{gathered}
0=\int_{\Gamma} z^{\nu} \frac{\Psi_{j+1}(z)}{q_{j+2}(z)} d z=\int_{\Gamma} \frac{z^{\nu}}{q_{j+2}(z)} \int_{\Delta_{j+1}} \frac{\Psi_{j}(x)}{z-x} d s_{r_{j}}^{j}(x) d z=\int_{\Delta_{j+1}} \Psi_{j}(x) \int_{\Gamma} \frac{z^{\nu}}{q_{j+2}(z)} \frac{d z}{z-x} d s_{r_{j}}^{j}(x)= \\
2 \pi i \int_{\Delta_{j+1}} x^{\nu} \Psi_{j}(x) \frac{d s_{r_{j}}^{j}(x)}{q_{j+2}(x)}, \quad \nu=0, \ldots, n_{r_{j}}^{j}+\operatorname{deg} q_{j+2}-1, \quad j=0, \ldots, m-1 .
\end{gathered}
$$

Since $n_{r_{j}}^{j}+\operatorname{deg} q_{j+2} \geq n_{r_{j}}^{j}+\left|n^{j+1}\right|=\left|n^{j}\right|$, we obtain (19).

In order to prove the last statement, let us assume that for some $j=0, \ldots, m-1$, we have that $\operatorname{deg} q_{j+2}>\left|n^{j+1}\right|$. From (20) it follows that $\Psi_{j}$ has at least $\left|n^{j}\right|+1$ sign changes on $\Delta_{j+1}$. This means that $\operatorname{deg} q_{j+1}>\left|n^{j}\right|$. Continuing downwards on the index $j$ we obtain that $\Psi_{0}$ has more than $\left|n^{0}\right|$ sign changes on $\Delta_{1}$. But this is impossible since $\Psi_{0}$ changes sign on $\Delta_{1}$ exactly at the $\left|n^{0}\right|$ zeros of $Q$. With this we conclude the proof.

REmark . Notice that $\mathbb{Z}_{+}^{1}=\mathbb{Z}_{+}^{1}(*)$ and $\mathbb{Z}_{+}^{2}=\mathbb{Z}_{+}^{2}(*)$. For $m=3$ the only indices left out in $\mathbb{Z}_{+}^{3}(*)$ are those strictly increasing $\left(n_{1}<n_{2}<n_{3}\right)$. For those indices it is also possible to prove analogues of Theorems 2 and 3 using the type of transformation employed in [4] to solve strong normality for such multi-indices. 


\section{VeCtor EQUilibrium PROBlem in the PRESENCE OF AN EXTERNAL FIELD}

Let

(1) $\Delta=\left(\Delta_{1}, \cdots, \Delta_{m}\right)$ be a set of $m$ closed bounded intervals of the real line (which do not reduce to points) and $m \in \mathbb{N}$;

(2) $\theta=\left(\theta_{1}, \ldots, \theta_{m}\right) \in \mathbb{R}_{+}^{m}, \mathbb{R}_{+}=(0,+\infty)$, and denote $|\theta|=\sum_{i=1}^{m} \theta_{i}$;

(3) $A=\left\|a_{i, j}\right\|$, is a real $(m \times m)$ symmetric matrix which is positive definite and $a_{i, j} \geq 0$, if $\Delta_{i} \cap \Delta_{j} \neq \emptyset$;

(4) $f=\left(f_{1}, \cdots, f_{m}\right)$, where $f_{i}, i=1, \cdots m$, is a real lower semi-continuous function on $\Delta_{i}$.

Let $\sigma$ be a finite positive Borel measure with compact support $\operatorname{supp}(\sigma)$ contained in $\mathbb{R}$ and $|\sigma|$ its total variation. For each $i=1, \cdots m, \mathcal{M}_{\theta_{i}}\left(\Delta_{i}\right)$ is the set of all positive Borel measures $\sigma$ such that $\operatorname{supp}(\sigma) \subset \Delta_{i}$ and $|\sigma|=\theta_{i}$. By $\mathcal{M}_{\theta}(\Delta)$ we denote the product $\mathcal{M}_{\theta}(\Delta)=\prod_{i=1}^{m} \mathcal{M}_{\theta_{i}}\left(\Delta_{i}\right)$.

For $\mu=\left(\mu_{1}, \ldots, \mu_{m}\right) \in \mathcal{M}_{\theta}(\Delta)$ we define the vector function

$$
W^{\mu}(z)=\left(V^{A \mu}+f\right)(z)=\int \ln \frac{1}{|z-x|} d A \mu(x)+f(z), \quad z \in \mathbb{C} .
$$

The $i$-th component of $W^{\mu}$ is given by

$$
W_{i}^{\mu}=\sum_{j=1}^{m} a_{i, j} V^{\mu_{j}}+f_{i}, \quad i=1, \cdots m
$$

where $V^{\mu_{j}}$ is the logarithmic potential of the measure $\mu_{j}$,

$$
V^{\mu_{j}}(z)=\int \ln \frac{1}{|z-x|} d \mu_{j}(x), \quad z \in \mathbb{C} .
$$

We define the mutual energy of two vector measures $\mu^{1}, \mu^{2} \in \mathcal{M}_{\theta}(\Delta)$ in the presence of $f$ to be

$$
J\left(\mu^{1}, \mu^{2}\right)=\sum_{i, j=1}^{m} \iint\left[a_{i, j} \ln \frac{1}{|z-x|}+\frac{f_{i}(z)+f_{j}(x)}{|\theta|}\right] d \mu_{i}^{1}(z) d \mu_{j}^{2}(x) .
$$

The energy of the vector measure $\mu \in \mathcal{M}_{\theta}(\Delta)$ can be written as

$$
J(\mu)=(A \mu, \mu)+2 \int f d \mu=\sum_{i, j=1}^{m} a_{i, j} I\left(\mu_{i}, \mu_{j}\right)+2 \sum_{i=1}^{m} \int f_{i}(x) d \mu_{i}(x),
$$

where

$$
I\left(\mu_{i}, \mu_{j}\right)=\iint \ln \frac{1}{|z-x|} d \mu_{i}(z) d \mu_{j}(x) .
$$

These formulas may be rewritten as

$$
J\left(\mu^{1}, \mu^{2}\right)=\int W^{\mu^{2}}(z) d \mu^{1}(z)+\int f(x) d \mu^{2}(x) .
$$

and

$$
J(\mu)=\int\left(W^{\mu}+f\right)(z) d \mu(z)
$$

where

$$
\int W^{\mu^{2}}(z) d \mu^{1}(z)=\sum_{i=1}^{m} \int W_{i}^{\mu^{2}}(z) d \mu_{i}^{1}(z), \quad \int f(x) d \mu^{2}(x)=\sum_{j=1}^{m} \int f_{j}(x) d \mu_{j}^{2}(x) .
$$

These concepts may be interpreted in electrostatic terms. $\Delta$ is a system of charge conductors where on each conductor $\Delta_{i}$ acts an external field $f_{i}, i=1, \cdots m$. The vector $\theta$ characterizes how much charge is allowed on each conductor. The measure $\mu \in \mathcal{M}_{\theta}(\Delta)$ gives the distribution of the charges. The entry $a_{i, j}$ of the matrix $A$ represents the law of interaction between the conductors $\Delta_{i}, \Delta_{j}, i, j=1, \ldots, m$.

For each $\mu \in \mathcal{M}_{\theta}(\Delta)$, we define

$$
w_{i}^{\mu}=\min _{x \in \Delta_{i}} W_{i}^{\mu}(x), \quad i=1, \ldots, m .
$$


In the sequel, we will assume that $f$ is such that there exists $\mu \in \mathcal{M}_{\theta}(\Delta)$ with $I\left(\mu_{i}, \mu_{i}\right)<+\infty, i=$ $1, \ldots, m$, and

$$
\int f(x) d \mu(x)<+\infty
$$

THEOREM 4. Each of the following problems (1) - (3) has a unique solution $\bar{\mu} \in \mathcal{M}_{\theta}(\Delta)$; the solution of all these problems is the same.

(1) $J(\bar{\mu})=\min _{\mu \in \mathcal{M}_{\theta}(\Delta)} J(\mu)$,

(2) $W_{i}^{\bar{\mu}}(x)=w_{i}^{\bar{\mu}}, \quad x \in \operatorname{supp}\left(\bar{\mu}_{i}\right), \quad i=1, \ldots, m$,

(3) $\max _{\mu \in \mathcal{M}^{i}(\bar{\mu})} w_{i}^{\mu}=w_{i}^{\bar{\mu}}, \quad i=1, \ldots, m$,

where

$$
\mathcal{M}^{i}(\bar{\mu})=\left\{\mu \in \mathcal{M}_{\theta}(\Delta): \mu_{j}=\bar{\mu}_{j}, j=1, \ldots, m, j \neq i\right\} .
$$

The measure $\bar{\mu}=\bar{\mu}(\Delta, \theta, A, f)$ is called extremal or equilibrium measure with respect to the initial data $(\Delta, \theta, A, f)$. For applications, the second statement is the most important. This part may be restated as follows: there exists a unique measure $\bar{\mu} \in \mathcal{M}_{\theta}(\Delta)$ and certain constants $w_{i}$, such that $W_{i}^{\bar{\mu}}(x)=w_{i}$ on $\operatorname{supp}\left(\bar{\mu}_{i}\right)$ and $W_{i}^{\bar{\mu}}(x) \geq w_{i}$ on $\Delta_{i}, i=1, \ldots, m$. Theorem 4 extends to the vector case a known result concerning the existence of an equilibrium measure in the presence of an external field. The vector case without external field has been treated in [6] and [12]. Our proof for the general case follows the scheme proposed in [12]. A similar result also holds for general regular compacts sets in the complex plane.

Of particular interest is the scalar case $(m=1)$ which we state in the form of a corollary, since it formally follows from the statement above, but will be used in proving Theorem 4 . Here $\Delta=\Delta_{1}, \theta>0, A=a>0, f$ is a lower semi-continuous function on $\Delta, W^{\mu}=a V^{\mu}+f$, and $w^{\mu}=\min _{x \in \Delta} W^{\mu}(x)$.

COROLlary 1. Each of the following problems (1) - (3) has a unique solution $\bar{\mu} \in \mathcal{M}_{\theta}(\Delta)$; the solution of all these problems is the same.

(1') $J(\bar{\mu})=\min _{\mu \in \mathcal{M}_{\theta}(\Delta)} J(\mu)$,

(2') $a V^{\bar{\mu}}+f=w^{\bar{\mu}}, \quad x \in \operatorname{supp}(\bar{\mu})$,

(3') $\max _{\mu \in \mathcal{M}_{\theta}(\Delta)} w^{\mu}=w^{\bar{\mu}}$.

Before proving Theorem 4 we need some auxiliary results.

Lemma 1. The functionals (21) and (22) are lower semi-continuous in the weak star topology of $\mathcal{M}_{\theta}(\Delta)$.

Proof. It is sufficient to show that the mutual energy is lower semi-continuous since $J(\mu)=$ $J(\mu, \mu)$. Since the components of $f$ are lower semi-continuous functions, it is easy to prove that each one of the integrals associated with these components in (21) defines a lower semi-continuous functional on $\mathcal{M}_{\theta_{i}}\left(\Delta_{i}\right)$ respectively. Therefore, it is sufficient to prove the lower semi-continuity property for the $\operatorname{sum} \sum_{i, j=1}^{m} a_{i, j} I\left(\mu_{i}^{1}, \mu_{j}^{2}\right)$.

Let us separate this sum in two. $\sum_{1}$ contains all the terms for which $a_{i, j} \geq 0$, and $\sum_{2}$ the rest which have $a_{i, j}<0$. By Theorem 2.1 page 168 in [12] each term of the first sum is lower semi-continuous and, therefore, so is $\sum_{1}$. In the second sum all the terms are continuous because by assumption if $a_{i, j}<0$ then $\Delta_{i} \cap \Delta_{j}=\emptyset$ and, hence, the logarithmic kernel is continuous on the compact set $\Delta_{i} \times \Delta_{j}$

Let us consider the following problem. Find

$$
J(\bar{\mu})=w=\inf \left\{J(\mu): \mu \in \mathcal{M}_{\theta}(\Delta)\right\}
$$

and the extremal measure $\bar{\mu}$ for which the infimum is attained.

Since the intervals $\Delta_{i}, i=1, \ldots, m$, do not reduce to points they have positive logarithmic capacity. Therefore there exist vector measures $\mu=\left(\mu_{1}, \ldots, \mu_{m},\right)$ whose components have finite energy

$$
I\left(\mu_{i}\right)=I\left(\mu_{i}, \mu_{i}\right)=\iint \ln \frac{1}{|z-x|} d \mu_{i}(z) d \mu_{i}(x)<+\infty, \quad i=1, \ldots, m .
$$


By assumption, for at least one such vector measure $\int f d \mu<+\infty$. Therefore,

$$
w=\inf \left\{J(\mu): \mu \in \mathcal{M}_{\theta}(\Delta)\right\}=\inf \left\{J(\mu): \mu \in \mathcal{M}_{\theta}^{*}(\Delta)\right\}<+\infty,
$$

where

$$
\mathcal{M}_{\theta}^{*}(\Delta)=\left\{\mu \in \mathcal{M}_{\theta}(\Delta): I\left(\mu_{i}\right)<\infty, i=1, \ldots, m\right\}
$$

Let

$$
\mathcal{M}^{*}(\Delta)=\bigcup_{\theta \in \mathbb{R}_{+}^{m}} \mathcal{M}_{\theta}^{*}(\Delta)
$$

Lemma 2. The extremal measure $\bar{\mu}$ in $\mathcal{M}_{\theta}^{*}(\Delta)$ exists and is unique. This is the unique measure satisfying

$$
\int W^{\bar{\mu}} d(\mu-\bar{\mu}) \geq 0, \quad \mu \in \mathcal{M}_{\theta}^{*}(\Delta) .
$$

Proof. The existence of $\bar{\mu}$ is an immediate consequence of the weak star compactness of $\mathcal{M}_{\theta}(\Delta)$ and the lower semi-continuity of the functional (22).

Set

$$
I\left(\mu^{1}, \mu^{2}\right)=\sum_{i, j=1}^{m} a_{i, j} I\left(\mu_{i}^{1}, \mu_{j}^{2}\right) .
$$

This quantity represents the mutual energy of the vector measures $\mu^{1}, \mu^{2}$ in the absence of an external vector field. Let $\mu=\left(\mu_{1}, \ldots, \mu_{m}\right)$ be a vector charge; that is, each component of $\mu$ is a signed measure (charge). It is well known that each $\mu_{i}$ admits a unique decomposition

$$
\mu_{i}=\mu_{i,+}-\mu_{i,-}, \quad i=1, \ldots, m .
$$

into two (positive) measures $\mu_{i,+}, \mu_{i,-}$. Let us assume that $\mu_{i,+}, \mu_{i,-}$, have finite energy, then there is no problem in defining $I\left(\mu_{i}, \mu_{j}\right)$ according to $(21)$. In fact,

$$
I\left(\mu_{i}, \mu_{j}\right)=I\left(\mu_{i,+}, \mu_{j,+}\right)+I\left(\mu_{i,-}, \mu_{j,-}\right)-I\left(\mu_{i,+}, \mu_{j,-}\right)-I\left(\mu_{i,-}, \mu_{j,+}\right) .
$$

Therefore, if $\mu^{1}, \mu^{2}$ are two vector charges whose components decompose into positive measures with finite energy we can define for them their mutual energy through formula (28). Set

$$
\widetilde{\mathcal{M}^{*}}(\Delta)=\left\{\mu=\mu^{1}-\mu^{2}: \mu^{1}, \mu^{2} \in \mathcal{M}^{*}(\Delta)\right\}
$$

Obviously, $\widetilde{\mathcal{M}}^{*}(\Delta)$ is the vector space of all vector charges whose components decompose into measures with finite energy.

Let $0 \leq \epsilon \leq 1$. If $\mu \in \mathcal{M}_{\theta}^{*}(\Delta)$ then $\widetilde{\mu}=\epsilon \mu+(1-\epsilon) \bar{\mu} \in \mathcal{M}_{\theta}^{*}(\Delta)$. Therefore,

$$
J(\widetilde{\mu})-J(\bar{\mu}) \geq 0
$$

or, equivalently (see (22)),

$$
I(\widetilde{\mu})+2 \int f d \widetilde{\mu}-I(\bar{\mu})-2 \int f d \bar{\mu} \geq 0 .
$$

Substituting $\widetilde{\mu}$ by its expression in terms of $\mu$ and $\bar{\mu}$, we have

$$
\epsilon^{2} I(\mu-\bar{\mu})+2 \epsilon I(\mu-\bar{\mu}, \bar{\mu})+2 \epsilon \int f d(\mu-\bar{\mu}) \geq 0,
$$

therefore,

$$
J(\widetilde{\mu})-J(\bar{\mu})=\epsilon^{2} I(\mu-\bar{\mu})+2 \epsilon \int W^{\bar{\mu}} d(\mu-\bar{\mu}) \geq 0 .
$$

Dividing by $\epsilon$ and letting $\epsilon$ tend to zero, it follows that

$$
\int W^{\bar{\mu}} d(\mu-\bar{\mu}) \geq 0 \text {. }
$$

which is $(27)$.

Making an appropriate change of variables, it is easy to verify that there is no loss of generality in the proof if we restrict to the case when

$$
\max \left\{|z|: z \in \Delta_{i}, i=1, \ldots, m\right\}<1
$$


Propositión 4.2, page 178, in [12] (here, the assumptions on $A$ are used) states that if (30) takes place, then $I(\mu)=I(\mu, \mu) \geq 0$ for all $\mu \in \widetilde{\mathcal{M}}^{*}(\Delta)$ and $I(\mu)=0$ if and only if $\mu \equiv 0$.

Let us assume that $\mu$ is another vector extremal measure. Take $\epsilon=1$. We have that $J(\widetilde{\mu})=$ $J(\mu)=J(\bar{\mu})$. From $(29)$ we deduce that

$$
0=I(\mu-\bar{\mu})+2 \int W^{\bar{\mu}} d(\mu-\bar{\mu}),
$$

which together with $(27)$ implies that $I(\mu-\bar{\mu}) \leq 0$. Hence, $I(\mu-\bar{\mu})=0$ and, consequently, $\mu \equiv \bar{\mu}$, as we needed to prove.

Now, let us assume that a certain vector measure $\bar{\lambda} \in \mathcal{M}_{\theta}^{*}(\Delta)$ satisfies

$$
\int W^{\bar{\lambda}} d(\lambda-\bar{\lambda}) \geq 0, \quad \lambda \in \mathcal{M}_{\theta}^{*}(\Delta)
$$

Proceeding as above, we obtain

$$
J(\lambda)-J(\bar{\lambda})=I(\lambda-\bar{\lambda})+2 \int W^{\bar{\lambda}} d(\lambda-\bar{\lambda}) \geq 0,
$$

since both terms on the right hand are non-negative. This implies that $\bar{\lambda}$ is the extremal measure and the proof is complete.

We say that a property holds quasi everywhere (q.e.) if it is true except on a set of capacity zero. For $\mu \in \mathcal{M}_{\theta}^{*}(\Delta)$, set

and

$$
J_{i}(\mu)=\int\left(W_{i}^{\mu}+f_{i}\right) d \mu_{i}
$$

$$
w_{i}:=\theta_{i}^{-1}\left[J_{i}(\bar{\mu})-\int f_{i} d \bar{\mu}_{i}\right]=\theta_{i}^{-1} \int W_{i}^{\bar{\mu}} d \bar{\mu}_{i} .
$$

Lemma 3. For each $j=1, \cdots m$,

$$
W_{j}^{\bar{\mu}}(x) \geq w_{j}, \quad \text { q.e. } \quad x \in \Delta_{j} .
$$

Proof. To the contrary, let us assume that for some $i$ there is a compact subset $K \subset \Delta_{i}$ with positive logarithmic capacity such that

$$
W_{i}^{\bar{\mu}}(x)<w_{i}, \quad x \in K
$$

Take an arbitrary measure $\mu_{i} \in \mathcal{M}_{\theta_{i}}^{*}(K)$. The existence of such a measure is guaranteed by the positivity of the logarithmic capacity of $K$. Consider the vector measure $\mu=\left(\mu_{1}, \cdots, \mu_{m}\right)$, where $\mu_{j}=\bar{\mu}_{j}, j \neq i$. Then

$$
\int W^{\bar{\mu}} d(\mu-\bar{\mu})=\int W_{i}^{\bar{\mu}} d\left(\mu_{i}-\bar{\mu}_{i}\right)<w_{i} \theta_{i}-w_{i} \theta_{i}=0
$$

which contradicts $(27)$.

Lemma 4. For each $j=1, \ldots m$,

$$
W_{j}^{\bar{\mu}}(x) \leq w_{j}, \quad x \in \operatorname{supp}\left(\bar{\mu}_{j}\right) .
$$

Proof. Suppose that for some $j$ there exists $x_{0} \in \operatorname{supp}\left(\bar{\mu}_{j}\right)$ such that $W_{j}^{\bar{\mu}}\left(x_{0}\right)>w_{j}$. Since $W_{j}^{\bar{\mu}}$ is lower semi-continuous it follows that there exists a neighborhood $\mathcal{V}$ of $x_{0}$ such that for all $x \in \mathcal{V}$ we have that $W_{j}^{\bar{\mu}}(x)>w_{j}$. On the other hand, $x_{0} \in \operatorname{supp}\left(\bar{\mu}_{j}\right)$; therefore, $\bar{\mu}_{j}(\mathcal{V})>0$.

Using Lemma 3 and that a measure of finite energy has measure zero on any set of capacity zero, we obtain

$$
\theta_{j} w_{j}=\int_{\Delta_{j}} W_{j}^{\bar{\mu}} d \bar{\mu}_{j}=\int_{\mathcal{V}} W_{j}^{\bar{\mu}} d \bar{\mu}_{j}+\int_{\Delta_{j} \backslash \mathcal{V}} W_{j}^{\bar{\mu}} d \bar{\mu}_{j}>w_{j} \bar{\mu}_{j}(\mathcal{V})+w_{j} \bar{\mu}_{j}\left(\Delta_{j} \backslash \mathcal{V}\right)=\theta_{j} w_{j},
$$

which is absurd.

Proof of Theorem 4. By Lemma 2 we know that problem 1 has a unique solution. Let us show that this solution solves problem 2 .

In fact, since an interval is a regular set in the sense of the solution of Dirichlet's problem, from (31) it follows that

$$
W_{j}^{\bar{\mu}}(x) \geq w_{j}, \quad x \in \Delta_{j}, \quad j=1, \ldots, m .
$$


Consequently, using (32), we obtain that

$$
W_{j}^{\bar{\mu}}(x)=w_{j}, \quad x \in \Delta_{j}^{*}=\operatorname{supp}\left(\bar{\mu}_{j}\right), \quad j=1, \ldots, m .
$$

Therefore, $w_{j}=w_{j}^{\bar{\mu}}$ and $\bar{\mu}$ solves the second problem in Theorem 4 .

Let us show that any solution $\bar{\lambda}$ of problem 2 also solves problem 1 . Notice that

$$
\int W^{\bar{\lambda}} d \bar{\lambda}=\sum_{j=1}^{m} w_{j}^{\bar{\lambda}} \theta_{j}
$$

On the other hand, if $\lambda \in \mathcal{M}_{\theta}^{*}(\Delta)$ since $W_{j}^{\bar{\lambda}} \geq w_{j}^{\bar{\lambda}}, x \in \Delta_{j}, j=1, \ldots, m$, it follows that

$$
\int W^{\bar{\lambda}} d \lambda \geq \sum_{j=1}^{m} w_{j}^{\bar{\lambda}} \theta_{j}
$$

Hence, (27) takes place and by Lemma $2, \bar{\lambda}=\bar{\mu}$ as needed.

In order to solve problem 3 let us start with the scalar case. Of course, from what was proved above we know that problems 1' and 2' of the corollary are equivalent and have the same solution. Let us prove that problem 3 ' has as unique solution the equilibrium measure $\bar{\mu} \in \mathcal{M}_{\theta}(\Delta)$ in the presence of the external field $f$.

Since $\bar{\mu} \in \mathcal{M}_{\theta}(\Delta)$, obviously $\sup _{\mu \in \mathcal{M}_{\theta}(\Delta)} w^{\mu} \geq w^{\bar{\mu}}$. Let us assume that there exists $\mu \in \mathcal{M}_{\theta}(\Delta)$ such that

$$
w^{\mu} \geq w^{\bar{\mu}}
$$

and show that then $\mu=\bar{\mu}$. This would prove that the supremum in 3 ' is certainly attained and only by the equilibrium measure.

We know that $\left(a V^{\bar{\mu}}+f\right)(x)=w^{\bar{\mu}}, x \in \operatorname{supp} \bar{\mu} \subset \Delta$; therefore,

$$
a\left(V^{\bar{\mu}}-V^{\mu}\right)(x)=\left(a V^{\bar{\mu}}+f\right)(x)-\left(a V^{\mu}+f\right)(x) \leq 0, \quad x \in \operatorname{supp}(\bar{\mu}) .
$$

The function $a\left(V^{\bar{\mu}}-V^{\mu}\right)$ is subharmonic in $\overline{\mathbb{C}} \backslash \operatorname{supp}(\bar{\mu})$. Recall that $a>0$ and potentials are superharmonic everywhere and harmonic in the complement of their support.

On $\operatorname{supp}(\bar{\mu})$ the function $\left(a V^{\bar{\mu}}+f\right)$ is continuous since it takes a constant value on that set, and $\mathrm{f}$ is lower semi-continuous. Hence on $\operatorname{supp}(\bar{\mu}), a V^{\bar{\mu}}$ is upper semi-continuous but being a potential it is also lower semi-continuous; therefore, it is continuous on supp $\bar{\mu}$ and by the continuity principle for potentials it is continuous on all $\mathbb{C}$. This yields that $a\left(V^{\bar{\mu}}-V^{\mu}\right)(x)$ is upper semi-continuous on all $\mathbb{C}$. In particular, for $x \in \operatorname{supp}(\bar{\mu})$, because of $(33)$

$$
\limsup _{z \rightarrow x} a\left(V^{\bar{\mu}}-V^{\mu}\right)(z) \leq a\left(V^{\bar{\mu}}-V^{\mu}\right)(x) \leq 0 .
$$

By the maximum principle for subharmonic functions it follows that

$$
a\left(V^{\bar{\mu}}-V^{\mu}\right)(x) \leq 0, \quad x \in \overline{\mathbb{C}} .
$$

Moreover, $a\left(V^{\bar{\mu}}-V^{\mu}\right)(\infty)=0$; consequently,

$$
a\left(V^{\bar{\mu}}-V^{\mu}\right)(x) \equiv 0, \quad x \in \overline{\mathbb{C}} \backslash \operatorname{supp}(\bar{\mu}) .
$$

Taking limit as $z \rightarrow x \in \operatorname{supp}(\bar{\mu}), z \in \mathbb{C} \backslash \operatorname{supp} \bar{\mu}$, we obtain

$$
a\left(V^{\bar{\mu}}-V^{\mu}\right)(x) \geq \limsup _{z \rightarrow x} a\left(V^{\bar{\mu}}-V^{\mu}\right)(z) \geq 0, \quad x \in \operatorname{supp}(\bar{\mu}) .
$$

From all these relations, we have that

$$
a\left(V^{\bar{\mu}}-V^{\mu}\right)(z) \equiv 0, \quad z \in \overline{\mathbb{C}},
$$

from which follows that indeed $\mu=\bar{\mu}$. We have completed the proof of the corollary.

Following the proof of the scalar case, it is easy to verify that the equilibrium vector measure $\bar{\mu}=\left(\bar{\mu}_{1}, \ldots, \bar{\mu}_{m}\right)$ is a solution of problem 3 . Let $\bar{\lambda}=\left(\bar{\lambda}_{1}, \ldots, \bar{\lambda}_{m}\right)$ be any other solution of this problem. Fix $i \in\{1, \ldots, m\}$ and let $\lambda \in \mathcal{M}^{i}(\bar{\lambda})$. We have that

$$
W_{i}^{\lambda}=a_{i, i} V^{\lambda_{i}}+\sum_{j \neq i} a_{i, j} V^{\bar{\lambda}_{j}}+f_{i}=a_{i, i} V^{\lambda_{i}}+F_{i}
$$


and $F_{i}$ is lower semi-continuous on $\Delta_{i}$; therefore, $\bar{\lambda}_{i}$ is the solution of the scalar equilibrium problem in the presence of the external field $F_{i}$. Since for the scalar case problem 3 ' is equivalent to 2 ', this means that

$$
W_{i}^{\bar{\lambda}}(x)=w_{i}^{\bar{\lambda}}=\min _{t \in \Delta_{i}} W_{i}^{\bar{\lambda}}(t), \quad x \in \operatorname{supp}\left(\bar{\lambda}_{i}\right), \quad i=1, \ldots, m .
$$

Therefore, $\bar{\lambda}$ solves problem 2 and by the uniqueness of the solution of this problem we conclude that $\bar{\lambda}$ is the solution of the vector equilibrium problem which is what we wanted to prove.

\section{Asymptotic BehaVior of the SECOND TyPE FUnCtions}

In this section, we study the logarithmic asymptotic behavior of sequences of second type functions $\left\{\Psi_{n, j}\right\}$ along certain sequences $\Lambda \subset \mathbb{Z}_{+}^{m}(*)$ of multi-indices such that $|n| \rightarrow+\infty$. To this end we must consider the logarithmic asymptotic behavior of the sequences of monic polynomials $\left\{q_{n, j}\right\}, n \in \Lambda$, as defined in Theorem 2. In the sequel, we explicitly indicate the dependence of all quantities on the multi-index $n$.

Let us fix a probability distribution $p=\left(p_{1}, \cdots, p_{m}\right)$ with the property that there do not exist $1 \leq k<j<i \leq m$ such that $p_{k}<p_{j}<p_{i}$. Set $p^{0}=p$. Assume that $p^{j}=\left(p_{j+1}^{j}, \ldots, p_{m}^{j}\right)$ has been defined, where $j=0, \ldots, m-2$. Then,

$$
p^{j+1}=\left(p_{j+2}^{j+1}, \ldots, p_{m}^{j+1}\right)
$$

is the vector obtained from $p^{j}$ extracting the first component $p_{r_{j}}^{j}$ such that

$$
p_{r_{j}}^{j} \geq \max \left\{p_{k}^{j}: j+1 \leq k \leq m\right\} .
$$

We consider the vector equilibrium problem with the initial data given by:

(1) $\Delta=\left(\Delta_{1}, \ldots, \Delta_{m}\right)$ is a set of $m$ closed intervals contained in the real line such that $\Delta_{j} \cap \Delta_{j+1}=\emptyset, j=1, \ldots, m-1$.

(2) $\theta=\left(\theta_{1}, \ldots, \theta_{m}\right)$, where

$$
\theta_{j}=\left|p^{j-1}\right|, \quad j=1, \ldots, m .
$$

(3) $A=\left\|a_{j, k}\right\|$ is the interaction matrix for which $a_{k, k}=2, a_{j, k}=-1$, if $|j-k|=1$, and $a_{j, k}=0$ otherwise.

(4) $f=\left(f_{1}, \ldots, f_{m}\right)$, where $f_{1}$ is a real lower semi-continuous function on $\Delta_{1}$ such that there exists $\mu_{1} \in \mathcal{M}_{\theta_{1}}\left(\Delta_{1}\right)$ with $I\left(\mu_{1}, \mu_{1}\right)<+\infty$ for which $\int f_{1}(x) d \mu_{1}(x)<+\infty$ and $f_{i}=0, i=2, \ldots, m$, on $\Delta_{i}$.

By $\bar{\mu}$ we denote the extremal measure of the equilibrium problem given by Theorem 4 corresponding to this initial data.

Let $\Lambda \subset \mathbb{Z}_{+}^{m}(*)$ be a sequence of multi-indices $n=\left(n_{1}, \ldots, n_{m}\right)$ such that $|n| \rightarrow \infty$ and

$$
\lim _{n \in \Lambda} \frac{n_{k}}{|n|}=p_{k}>0, \quad k=1, \ldots m .
$$

Let $q_{n, j}=q_{j}, j=1, \ldots, m+1$, be the polynomials defined in (9). Recall that $q_{n, 1}=Q_{n}$ and $q_{n, m+1} \equiv 1$. Therefore, $q_{n, j}$ has simple zeros all lying in the interior of $\Delta_{j}$ and

$$
\operatorname{deg} q_{n, j}=\left|n^{j-1}\right|, \quad j=1, \ldots, m+1
$$

$\left(\left|n^{m}\right|=0\right)$. Notice that by assumption

$$
\lim _{n \in \Lambda} \frac{\left|n^{j-1}\right|}{|n|}=\theta_{j}, \quad j=1, \ldots m .
$$

Let $\chi(q)$ be the zero counting measure associated with the monic polynomial $q$. That is, $\chi(q)$ assigns measure 1 to each point which is a zero of $q$ (counting multiplicities) and measure zero to all other points.

We are given two sequences of monic polynomials $\left\{\alpha_{n}\right\},\left\{\beta_{n}\right\}, n \in \Lambda$, with real coefficients such that $\operatorname{deg} \beta_{n}=\kappa_{n}, \operatorname{deg} \alpha_{n} \leq|n|+\kappa_{n}+\min \left\{n_{1}, \ldots, n_{m}\right\}$. The zeros of $\beta_{n}$ have even multiplicity 
and lie on $\Delta_{1}$ and the zeros of $\alpha_{n}$ belong to a compact subset $E$ of $D=\overline{\mathbb{C}} \backslash \Delta_{1}$. We assume that there exist measures $\alpha, \beta$ with support contained in $E \subset D$ and $\Delta_{1}$ respectively such that

$$
\lim _{n \in \Lambda} \frac{1}{|n|} \chi\left(\alpha_{n}\right)=\alpha, \quad \lim _{n \in \Lambda} \frac{1}{|n|} \chi\left(\beta_{n}\right)=\beta .
$$

The convergence of measures is in the weak star sense.

Set

$$
f_{n, 1}(x)=\frac{1}{|n|}\left(V^{\chi\left(\beta_{n}\right)}(x)-V^{\chi\left(\alpha_{n}\right)}(x)\right) .
$$

By (36) and known properties of the potential (see [7] and [10]), it follows that:

- $f_{1}(x)=\lim _{n \in \Lambda} f_{n, 1}(x)=V^{\beta}(x)-V^{\alpha}(x), x \in \Delta_{1}$, where convergence is in measure on $\Delta_{1}$.

- Each $f_{n, 1}$ as well as $f_{1}$ is lower semi-continuous on $\Delta_{1}$.

- Each $f_{n, 1}$ and $f_{1}$ is weakly approximatively continuous on $\Delta_{1}$. A function $g$ is weakly approximatively continuous at $x_{0} \in \Delta_{1}$, if there exists a set $e\left(x_{0}\right) \subset \Delta_{1}$ of positive measure such that

$$
\liminf _{x \rightarrow x_{0}, x \in \Delta_{1}} g(x)=\lim _{x \rightarrow x_{0}, x \in e\left(x_{0}\right)} g(x)=g\left(x_{0}\right) .
$$

- $\lim _{n \rightarrow \infty} \min _{\Delta_{1}} f_{n, 1}(x)=\min _{\Delta_{1}} f_{1}(x)$.

This type of convergence will be denoted

$$
\mathcal{F}-\lim _{n \rightarrow \infty} f_{n, 1}=f_{1} .
$$

This function $f_{1}$ is taken for the first component of $f$ above. We must verify that (26) takes place. In fact, take any $\mu \in \mathcal{M}_{\theta}^{*}(\Delta)$ such that $\mu_{1}$ is the equilibrium measure on $\Delta_{1}$ in the absence of an external field. Then

$$
\int f(x) d \mu(x)=\int_{\Delta_{1}}\left(V^{\beta}-V^{\alpha}\right)(x) d \mu_{1}(x)=\int_{\Delta_{1}} V^{\mu_{1}}(x) d \beta(x)-\int_{\Delta_{1}} V^{\alpha}(x) d \mu_{1}(x)<\infty
$$

since $V^{\mu_{1}}$ and $V^{\alpha}$ are continuous on $\Delta_{1}$.

Let $\sigma$ be a finite positive Borel measure supported on a compact subset of the real line. We say that $\sigma \in \mathbf{R e g}$ if

$$
\lim _{l} \kappa_{l}^{1 / l}=\operatorname{Cap}(\operatorname{supp}(\sigma)),
$$

where $\kappa_{l}>0$ denotes the leading coefficient of the orthonormal polynomial of degree $l$ with respect to $\sigma$ and $\operatorname{Cap}(\cdot)$ the logarithmic capacity of the indicated set. For more details on this definition and properties of the so called class of regular measures see Chapter 2 in [13]. In particular, it is well known that $\sigma^{\prime}>0$ almost everywhere (on its support) implies that $\sigma \in \mathbf{R e g}$. Moreover, the class of regular measures is substantially larger than the class of measures for which $\sigma^{\prime}>0$ almost everywhere.

We have the following result which generalizes Theorems 3 to 5 from [8] in the case of Nikishin systems of measures. The proof follows the same scheme but taking into consideration the solution of the vector equilibrium problem in the presence of an external vector field.

THEOREM 5. Let us assume that $\sigma_{1}^{\prime}>0$ almost everywhere on $\Delta_{1}$, and $\sigma_{j} \in \mathbf{R e g}$ for $j=2, \ldots, m$, We also assume that (35) and (36) take place. For each $j=1, \ldots, m$, we have

$$
\lim _{n \in \Lambda} \frac{1}{|n|} \chi\left(q_{n, j}\right)=\bar{\mu}_{j} .
$$

Therefore,

$$
\lim _{n \in \Lambda}\left|q_{n, j}(z)\right|^{\frac{1}{n \mid}}=\exp \left(-V^{\bar{\mu}_{j}}(z)\right), \quad j=1, \ldots, m,
$$

uniformly on compact subsets of $\mathbb{C} \backslash \Delta_{j}$. Moreover,

$$
\lim _{n \in \Lambda}\left(\int q_{n, j}^{2} \frac{\left|\psi_{n, j-1}\right| d s_{r_{j-1}}^{j-1}}{\left|q_{n, j+1}\right|}\right)^{\frac{1}{|n|}}=e^{-v_{j}}, \quad j=1, \ldots, m .
$$


where $v_{j}=w_{1}^{\bar{\mu}}+\cdots+w_{j}^{\bar{\mu}}$, and

$$
\lim _{n \in \Lambda}\left|\Psi_{n, j}\right|^{\frac{1}{|n|}}=\exp \left(V^{\bar{\mu}_{j}}-V^{\bar{\mu}_{j+1}}-v_{j}\right) .
$$

uniformly on each compact subset of the complement of $\Delta_{j} \cup \Delta_{j+1} \cdot\left(\Delta_{m+1}=\emptyset, V^{\bar{\mu}_{m+1}} \equiv 0\right.$.)

Proof. Different multi-indices $n \in \Lambda \subset \mathbb{Z}_{+}^{m}(*)$ may have associated different $\Sigma^{j}$ and $r_{j}, j=$ $0, \ldots, m-1$. See the definitions in the beginning of Section 3. In particular, the measures $s_{r_{j}}^{j}, j=$ $0, \ldots, m-1$ may depend on $n$. For simplicity in the notation, we have not indicated this above. So far this has not been important because the preceding results were proved for fixed $n$, but now we are taking limit on $n$. Nevertheless, under the assumptions of theorem 5 this fact has no influence. Notice that according to the construction, there are only a finite number of possible systems $\Sigma^{j}, j=0, \ldots, m-1$, and measures $s_{r_{j}}^{j}, j=0, \ldots, m-1$, associated to different multiindices. We will assume that we have taken a subsequence of multi-indices $\Lambda^{\prime} \subset \Lambda$, with an infinite number of elements, such that for all $n \in \Lambda^{\prime}$ we obtain the same measures $s_{r_{j}}^{j}, j=0, \ldots, m-1$, and prove that (37)-(40) hold true. Since the right hand sides of (37)-(40) only depend on the initial data of the equilibrium problem, which is independent of the construction of the auxiliary systems $\Sigma^{j}, j=0, \ldots, m-1$, and $\Lambda$ may be partitioned into a finite number of $\Lambda^{\prime}$ plus a finite number of multi-indices, the result holds true as stated. In the sequel, without loss of generality, we will assume that all $n \in \Lambda$ have associated the same $\Sigma^{j}, j=0, \ldots, m-1$.

Using (9) and (19) it follows that

$$
\int_{\Delta_{j+1}} x^{\nu} q_{n, j+1}(x) \psi_{n, j}(x) \frac{d s_{r_{j}}^{j}(x)}{q_{n, j+2}(x)}=0, \quad \nu=0, \ldots,\left|n^{j}\right|-1, \quad j=0, \ldots m-1 .
$$

That is

$$
\int_{\Delta_{j+1}} x^{\nu} q_{n, j+1}(x) h_{n, j}(x) d s_{r_{j}}^{j}(x)=0, \quad \nu=0, \ldots,\left|n^{j}\right|-1, \quad j=0, \ldots m-1
$$

where

$$
h_{n, j}=\frac{\left|\psi_{n, j}\right|}{\left|q_{n, j+2}\right|}, \quad j=0, \ldots, m-1 .
$$

(Recall that $\psi_{n, j}$ and $q_{n, j+2}$ have constant sign on $\Delta_{j+1}$.)

The polynomial $q_{n, j+1}$ is orthogonal with respect to the varying measure

$$
d \sigma_{n, j+1}=h_{n, j} d s_{r_{j}}^{j}, \quad j=0, \ldots, m-1 .
$$

Define

$$
\mu_{n, j}=\frac{1}{|n|} \chi\left(q_{n, j}\right), \quad j=1, \ldots, m,
$$

and set $\mu_{n}=\left(\mu_{n, 1}, \ldots, \mu_{n, m}\right)$. Since the sequence $\left\{\mu_{n}\right\}, n \in \Lambda$, is weakly compact, in order to prove (37) it is sufficient to show that any convergent subsequence $\left\{\mu_{n}\right\}, n \in \Lambda^{\prime}, \Lambda^{\prime} \subset \Lambda$, satisfies

$$
\lim _{n \in \Lambda^{\prime}} \mu_{n}=\bar{\mu} .
$$

Therefore, without loss of generality, we can assume that there exists

$$
\lim _{n \in \Lambda} \mu_{n}=\mu=\left(\mu_{1}, \ldots, \mu_{m}\right) .
$$

and we must show that $\mu=\bar{\mu}$. To this end we make use of Theorem 1 in [7] and Theorem 3.3.3 in [13] related with the $n$th root asymptotic behavior of orthogonal polynomials with respect to varying measures. For convenience of the reader, we state the corresponding result in a combined statement. The result with weaker assumptions on the sequence of functions $\left\{g_{l}\right\}_{l \in \Lambda}$ follows [7] whereas the weaker assumptions on $\sigma$ correspond to [13].

THEOREM 6. Let $\Lambda \subset \mathbb{N}$. Suppose that a sequence of monic polynomials $\left\{q_{l}\right\}_{l \in \Lambda}$ satisfies the orthogonality relations

$$
\int x^{k} q_{l}(x) d \sigma_{l}(x)=0, \quad k=0, \ldots, \operatorname{deg} q_{l}-1, \quad l \in \Lambda,
$$


where

$$
d \sigma_{l}=\exp \left(-g_{l}\right) d \sigma
$$

Let us assume that either $\sigma^{\prime}>0$ almost everywhere on its support given by an interval $\Delta$, the functions in the sequence $\left\{g_{l}\right\}_{l \in \Lambda}$ as well as $g$ are lower semi-continuous functions on $\Delta$ and verify

$$
\mathcal{F}-\lim _{l \in \Lambda} \frac{1}{l} g_{l}(x)=g(x), \quad x \in \Delta,
$$

or $\sigma \in \mathbf{R e g}$, the functions in the sequence $\left\{g_{l}\right\}_{l \in \Lambda}$ as well as $g$ are continuous functions on $\Delta$ and satisfy

$$
\lim _{l \in \Lambda} \frac{1}{l} g_{l}(x)=g(x), \quad x \in \Delta,
$$

uniformly on $\Delta$. Assume that there exists

$$
\lim _{l \in \Lambda} \frac{\operatorname{deg} q_{l}}{l}=\theta
$$

Then

$$
\lim _{l \in \Lambda} \frac{1}{l} \chi\left(q_{l}\right)=\bar{\mu}
$$

and

$$
\lim _{l \in \Lambda}\left(\int q_{l}^{2} d \sigma_{l}(x)\right)^{\frac{1}{l}}=e^{-w}
$$

where $\bar{\mu}=\bar{\mu}(\Delta, \theta, 2, g)$ is the solution of the scalar equilibrium problem given by Corollary 1 with $a=2$, external field given by $g$, and $w=w^{\bar{\mu}}$ is the associated equilibrium constant.

From (42) it follows that

$$
\lim _{n \in \Lambda} \frac{1}{|n|} \log \left|q_{n, j}\right|=\lim _{n \in \Lambda} V^{\mu_{n, j}}=-V^{\mu_{j}},
$$

uniformly on each compact subset of $\mathbb{C} \backslash \Delta_{j}$. Since $\psi_{n, 0}=\frac{\beta_{n}}{\alpha_{n}}$, from (36) we get

$$
\mathcal{F}-\lim _{n \in \Lambda} \frac{1}{|n|} \log \left|\psi_{n, 0}\right|^{-1}=f_{1}
$$

on $\Delta_{1}$. Set

$$
2 V^{\mu_{1}}-V^{\mu_{2}}+f_{1}=W_{1}^{\mu} .
$$

Using (41) for $j=0$ and Theorem 6 (see (42), (44) and (45)), we obtain that $\mu_{1}$ satisfies the equilibrium conditions

$$
W_{1}^{\mu}(x)=\min _{\Delta_{1}} W_{1}^{\mu}=w_{1}^{\mu}, \quad x \in \operatorname{supp}\left(\mu_{1}\right)
$$

and

$$
-\lim _{n \in \Lambda} \frac{1}{|n|} \log \left(\int q_{n, 1}^{2} d \sigma_{n, 1}\right)=w_{1}^{\mu}
$$

From (41) it follows that

$$
q(z) \int \frac{q_{n, j+1}(x)}{z-x} \frac{\psi_{n, j}(x)}{q_{n, j+2}(x)} d s_{r_{j}}^{j}(x)=\int \frac{q(x) q_{n, j+1}(x)}{z-x} \frac{\psi_{n, j}(x)}{q_{n, j+2}(x)} d s_{r_{j}}^{j}(x)
$$

where $q$ is any polynomial of degree $\leq\left|n^{j}\right|$. If we use this formula for $q=q_{n, j+1}$ and $q=q_{n, j+2}$ respectively, we obtain

$$
\int \frac{q_{n, j+1}(x)}{z-x} \frac{\psi_{n, j}(x)}{q_{n, j+2}(x)} d s_{r_{j}}^{j}(x)=\frac{1}{q_{n, j+1}(z)} \int \frac{q_{n, j+1}^{2}(x)}{z-x} \frac{\psi_{n, j}(x)}{q_{n, j+2}(x)} d s_{r_{j}}^{j}(x)
$$

and (see also (7))

$$
\int \frac{q_{n, j+1}(x)}{z-x} \frac{\psi_{n, j}(x)}{q_{n, j+2}(x)} d s_{r_{j}}^{j}(x)=\frac{1}{q_{n, j+2}(z)} \int \frac{q_{n, j+2}(x) q_{n, j+1}(x)}{z-x} \frac{\psi_{n, j}(x)}{q_{n, j+2}(x)} d s_{r_{j}}^{j}(x)=\frac{\Psi_{n, j+1}(z)}{q_{n, j+2}(z)} .
$$


Therefore,

$$
\psi_{n, j}(z)=\frac{1}{q_{n, j}(z)} \int \frac{q_{n, j}^{2}(t)}{z-t} d \sigma_{n, j}(t), \quad z \in \mathbb{C} \backslash \Delta_{j}, \quad j=1 \ldots, m .
$$

Let us prove that for each $j=1, \ldots, m$, there exists the limit

$$
v_{j}=-\lim _{n \in \Lambda} \frac{1}{|n|} \log \left(\int q_{n, j}^{2} d \sigma_{n, j}\right) .
$$

We proceed by induction. We know that $v_{1}=w_{1}^{\mu}$ for $j=1$. Let us assume that the limit exists for some $j, 1 \leq j \leq m-1$ and show that the assertion is also true for $j+1$.

It is well known and easy to verify (see, for example, page 158 in [13]) that for each compact subset $\mathcal{K}$ of $\mathbb{C} \backslash \Delta_{j}$ there exist positive constants $C_{1}(\mathcal{K})$ and $C_{2}(\mathcal{K})$ such that

$$
C_{1}(\mathcal{K})\left|\int q_{n, j}^{2}(x) d \sigma_{n, j}(x)\right| \leq\left|\int \frac{q_{n, j}^{2}(x)}{z-x} d \sigma_{n, j}(x)\right| \leq C_{2}(\mathcal{K})\left|\int q_{n, j}^{2}(x) d \sigma_{n, j}(x)\right| . \quad z \in \mathcal{K} .
$$

From these inequalities and (47) it follows that

$$
-\lim _{n \in \Lambda} \frac{1}{|n|} \log \left|\psi_{n, j}(z)\right|=-V^{\mu_{j}}(z)+v_{j}
$$

uniformly on each compact subset of $\mathbb{C} \backslash \Delta_{j}$; in particular, on $\Delta_{j+1}$. Therefore,

$$
-\lim _{n \in \Lambda} \frac{1}{|n|} \log h_{n, j}=v_{j}-V^{\mu_{j+2}}-V^{\mu_{j}} .
$$

uniformly on $\Delta_{j+1}$. Using (41) and Theorem 6 for $j+1$ (notice that now it is only required that $\left.\sigma_{j+1} \in \mathbf{R e g}\right)$, it follows that $\mu_{j+1}$ satisfies the scalar equilibrium problem

$$
W_{j+1}^{\mu}(x)+v_{j}=2 V^{\mu_{j+1}}(x)-V^{\mu_{j+2}}(x)-V^{\mu_{j}}(x)+v_{j}=\min _{\Delta_{j+1}} W_{j+1}^{\mu}(x)+v_{j}=w_{j+1}^{\mu}+v_{j}
$$

for $x \in \operatorname{supp} \mu_{j+1}$, and

$$
v_{j+1}=-\lim _{n \in \Lambda} \frac{1}{|n|} \log \left(\int q_{n, j+1}^{2}(x) d \sigma_{n, j+1}(x)\right)=w_{j+1}^{\mu}+v_{j} .
$$

From (46) and (49) it follows that the vector measure $\mu$ satisfies the equilibrium conditions for all $j=1, \ldots, m$. Therefore, by Theorem 1 we have that $\mu=\bar{\mu}$ and thus obtained (37), (38), and (39). Formulas (38) and (48) yield (40) and we conclude the proof of Theorem 5.

REMARK . If $\beta_{n} \equiv 1, n \in \Lambda$ (that is, we do not fix poles), then $\lim _{n \in \Lambda} f_{n, 1}=f_{1}$ uniformly on $\Delta_{1}$ and $f_{1}$ is continuous on $\Delta_{1}$. In this situation we can also use Theorem 3.3.3 in [13] for the initial step in the proof of Theorem 5 replacing the condition $\sigma_{1}^{\prime}>0$ a. e. on $\Delta_{1}$ by the much weaker one $\sigma_{1} \in \mathbf{R e g}$. If this is the case, regarding the conditions on the measures, in Theorem 5 it is sufficient to assume that $\sigma_{j} \in \mathbf{R e g}, j=1, \ldots, m$.

\section{RAte of CONVERGENCE OF GHPA}

Let $S=\left(s_{1}, \ldots, s_{m}\right)=\mathcal{N}\left(\sigma_{1}, \ldots, \sigma_{m}\right)$ be a Nikishin system of measures and $\widehat{S}=\left(\widehat{s}_{1}, \ldots, \widehat{s}_{m}\right)$ the corresponding Nikishin system of functions. We will assume that $\sigma_{j}^{\prime}>0$ almost everywhere on $\Delta_{j}, j=1, \ldots, m$, although this assumption may be weakened as explained in the last remark. Let $\Lambda \subset \mathbb{Z}_{+}^{m}(*)$ be such that (35) takes place. For each $n \in \Lambda$, let $R_{n}=\left(R_{n, 1}, \ldots, R_{n, m}\right)$ be the GHPA associated with $\widehat{S}$ with respect to the monic polynomials $\alpha_{n}, \beta_{n}$ as indicated in Section 1 , where the sequences $\left\{\alpha_{n}\right\},\left\{\beta_{n}\right\}, n \in \Lambda$, satisfy (36). The object of this section is to study the rate of convergence of $\left\{R_{n, j}\right\}, n \in \Lambda$, to $\widehat{s}_{j}, j=1, \ldots m$. For this purpose, we use an integral formula for the remainder and the asymptotic formulas obtained in Theorem 5 .

According to ii) the function on the left has a zero of order at least one at infinity and is holomorphic in the complement of $\Delta_{1}$. Integrating along a close curve $\Gamma$ with winding number 1 for all its interior points such that the zeros of $\alpha_{n}$ and $z$ lie in the exterior of $\Gamma$, and $\Delta_{1}$ is surrounded by $\Gamma$, we obtain

$$
\left(\frac{\beta_{n} Q_{n} \widehat{s}_{j}-P_{n, j}}{\alpha_{n}}\right)(z)=\frac{1}{2 \pi i} \int_{\Gamma}\left(\frac{\beta_{n} Q_{n} \widehat{s}_{j}-P_{n, j}}{\alpha_{n}}\right)(\zeta) \frac{d \zeta}{z-\zeta}=\frac{1}{2 \pi i} \int_{\Gamma}\left(\frac{\beta_{n} Q_{n} \widehat{s}_{j}}{\alpha_{n}}\right)(\zeta) \frac{d \zeta}{z-\zeta} .
$$


Substituting $\widehat{s}_{j}$ by its integral expression and using Fubini's Theorem, for each $j=1, \ldots, m$, we have

$$
\delta_{n, j}(z)=\left(\widehat{s}_{j}-R_{n, j}\right)(z)=\frac{\left(\alpha_{n} \Phi_{n, j}\right)(z)}{\left(\beta_{n} Q_{n}\right)(z)}, \quad z \in D=\mathbb{C} \backslash \Delta_{1}, \quad j=1, \ldots, m,
$$

where

$$
\Phi_{n, j}(z)=\int_{\Delta_{1}} \frac{\left(\beta_{n} Q_{n}\right)(x)}{\alpha_{n}(x)} \frac{d s_{j}(x)}{z-x} .
$$

By Theorem 5 (recall that $q_{n, 1}=Q_{n}$ ) and (36) we know that

$$
\lim _{n \in \Lambda}\left|\frac{\alpha_{n}}{\beta_{n} Q_{n}}\right|^{\frac{1}{|n|}}=\exp \left(V^{\bar{\mu}_{1}}+f_{1}\right)
$$

uniformly on each compact subset of the region $D \backslash E=\mathbb{C} \backslash\left(E \cup \Delta_{1}\right)$. On compact subsets of $D$ the same holds taking upper limit instead of limit in (51). Therefore, the problem reduces to finding the limit of $\left\{\Phi_{n, j}\right\}, n \in \Lambda, j=1, \ldots, m$. To this end we must establish the connection between the functions $\Phi_{n, j}$ and $\Psi_{n, j}$.

We shall do this in two steps. First, we see the relation between $\Psi_{n, j}$ and the remainder of an auxiliary Nikishin system of functions. Then, we compare the remainder functions of the two Nikishin systems. Let us introduce the auxiliary Nikishin system.

To each $n \in \mathbb{Z}_{+}^{m}$ we can associate a permutation $\tau=\tau_{n}$ of $\{1, \ldots, m\}$ as follows. For each $i \in\{1, \ldots, m\}$

$$
\tau(i)=j \quad \text { if } \quad\left\{\begin{array}{ll}
n_{j} \geq n_{k} & \text { for } k>j \\
n_{j}>n_{k} & \text { for } k<j
\end{array}\right\} \quad \text { for all } \quad k \in\{1, \ldots, m\} \backslash\{\tau(1), \ldots, \tau(i-1)\} .
$$

When $n \in \mathbb{Z}_{+}^{m}(*)$ there do not exist $1 \leq i<j<k \leq m$ such that $n_{i}<n_{j}<n_{k}$. In terms of $\tau$ this means that there do not exist $1 \leq i<j<k \leq m$ such that $\tau(i)>\tau(j)>\tau(k)$. In other words, $\{1, \ldots, m\}$ may be partitioned into two subsets on which $\tau$ is increasing. We say that $\tau \in S_{m}(*)$ if this property holds true. Obviously, each $\tau \in S_{m}(*)$ is associated to infinitely many multi-indices in $\mathbb{Z}_{+}^{m}(*)$.

Let $\Lambda \subset \mathbb{Z}_{+}^{m}(*)$. To each $n \in \Lambda$ we associate the multi-index $\widetilde{n}=\left(n_{r_{0}}^{0}, \ldots, n_{r_{m-1}}^{m-1}\right)$. It is easy to verify that $\widetilde{n}=\left(n_{\tau(1)}, \ldots, n_{\tau(m)}\right)$. Moreover, $\tau(j)=r_{j-1}-d(j)$ where $d(j)$ is equal to the number of $i, 0 \leq i<j-1$, such that $r_{i}>\tau(j)$. In principle, the permutation $\tau$ depends on $n \in \Lambda$, but for simplicity we will not indicate it. Set $\widetilde{\Lambda}=\{\widetilde{n}: n \in \Lambda\}$.

The auxiliary Nikishin system is $\left(\vartheta_{1}, \ldots, \vartheta_{m}\right)$ where $\vartheta_{j}=s_{r_{j-1}}^{j-1}, j=1, \ldots, m$. The measures $s_{r_{j-1}}^{j-1}$ are the ones defined in relation $(7)$ of Section 3. Set $\left(\vartheta_{1,1}, \ldots, \vartheta_{1, m}\right)=\mathcal{N}\left(\vartheta_{1}, \ldots, \vartheta_{m}\right)$. Let $\widetilde{R}_{\widetilde{n}}=\left(\frac{\widetilde{P}_{\tilde{\widetilde{Q}}, 1}}{\widetilde{\widetilde{Q}}_{\tilde{n}}}, \ldots, \frac{\widetilde{P}_{\tilde{\tilde{Q}}, m}}{\widetilde{Q}_{\tilde{n}}}\right)$ be the sequence of GHPA corresponding to the system of functions $\left(\widehat{\vartheta}_{1,1}, \ldots \widehat{\vartheta}_{1, m}\right)$, the sequence of multi-indices $\widetilde{\Lambda}$, and the sequences of polynomials $\alpha_{n}$ and $\beta_{n}$ (the same polynomials as for the initial system considering the correspondence $n \leftrightarrow \widetilde{n}$ ).

In Theorem 1 of [1] it was proved that the common denominator $\widetilde{Q}_{\widetilde{n}}$ of $\widetilde{R}_{\widetilde{n}}$ satisfies the same orthogonality relations as the common denominator $Q_{n}$ of $R_{n}$. Therefore, $\widetilde{Q}_{\widetilde{n}}=Q_{n}$. Moreover, the functions $\Psi_{n, j}$ defined for the initial Nikishin system equal the corresponding ones for the auxiliary Nikishin system which we have just introduced; therefore, both generate the same polynomials $q_{n, j}, j=1, \ldots, m$.

From (50) applied to the auxiliary Nikishin system, we have that

$$
\widetilde{\delta}_{n, j}(z)=\left(\widehat{\vartheta}_{1, j}-\widetilde{R}_{\widetilde{n}, j}\right)(z)=\frac{\left(\alpha_{n} \widetilde{\Phi}_{\widetilde{n}, j}\right)(z)}{\left(\beta_{n} Q_{n}\right)(z)}, \quad z \in D=\mathbb{C} \backslash \Delta_{1}, \quad j=1, \ldots, m,
$$

where

$$
\widetilde{\Phi}_{\tilde{n}, j}(z)=\int_{\Delta_{1}} \frac{\left(\beta_{n} Q_{n}\right)(x)}{\alpha_{n}(x)} \frac{d \vartheta_{1, j}(x)}{z-x}=\int_{\Delta_{1}} \cdots \int_{\Delta_{j}} \frac{\left(\beta_{n} Q_{n}\right)\left(x_{1}\right)}{\alpha_{n}\left(x_{1}\right)} \frac{d \vartheta_{1}\left(x_{1}\right) d \vartheta_{2}\left(x_{2}\right) \cdots d \vartheta_{j}\left(x_{j}\right)}{\left(z-x_{1}\right)\left(x_{1}-x_{2}\right) \cdots\left(x_{j-1}-x_{j}\right)} .
$$


LEMma 5. Assume that the same permutation $\tau$ is associated to all $n \in \Lambda \subset \mathbb{Z}_{+}^{m}(*)$. For each $j=1,2, \ldots, m$, we have

$$
\widetilde{\Phi}_{\tilde{n}, j}(z)=(-1)^{j-1} \Psi_{n, j}(z)+\sum_{k=1}^{j-1} u_{j, k}(z) \Psi_{n, k}(z), \quad z \in \mathbb{C} \backslash\left(\bigcup_{k=1}^{j} \Delta_{k}\right),
$$

where the functions $u_{j, k}$ are analytic in $\mathbb{C} \backslash\left(\bigcup_{k=1}^{j} \Delta_{k}\right)$ and do not depend on $n$. For each $j=$ $1,2, \ldots, m$

$$
\Phi_{n, \tau(j)}(z)=\widetilde{\Phi}_{\widetilde{n}, j}(z)+\sum_{k=1}^{j-1} \ell_{j, k}(z) \widetilde{\Phi}_{\widetilde{n}, k}(z)+\ell_{j, 0}(z), \quad z \in \mathbb{C} \backslash \Delta_{k},
$$

where $\ell_{j, k}, k=0, \ldots, j-1$, denote polynomials that do not depend on $n \in \Lambda$ of degree $\leq m-1$. If $\min _{1 \leq i \leq m} n_{i} \geq m-1, n \in \Lambda$, then $\ell_{j, 0} \equiv 0$.

Proof. We begin proving (53). From the definition

$$
\Psi_{n, j}(z)=\int_{\Delta_{1}} \cdots \int_{\Delta_{j}} \frac{\left(\beta_{n} Q_{n}\right)\left(x_{1}\right)}{\alpha_{n}\left(x_{1}\right)} \frac{d \vartheta_{1}\left(x_{1}\right) d \vartheta_{2}\left(x_{2}\right) \cdots d \vartheta_{j}\left(x_{j}\right)}{\left(x_{2}-x_{1}\right) \cdots\left(x_{j}-x_{j-1}\right)\left(z-x_{j}\right)} .
$$

For $j=1, \widetilde{\Phi}_{\widetilde{n}, j}(z)=\Psi_{n, j}(z)\left(=\Phi_{n, r_{0}}\right)$. When $2 \leq j \leq m$, we have

$$
\widetilde{\Phi}_{\tilde{n}, j}(z)+(-1)^{j} \Psi_{n, j}(z)=\int_{\Delta_{1}} \cdots \int_{\Delta_{j}} \frac{\left(\beta_{n} Q_{n}\right)\left(x_{1}\right)}{\alpha_{n}\left(x_{1}\right)} \frac{\left(x_{1}-x_{j}\right) d \vartheta_{1}\left(x_{1}\right) d \vartheta_{2}\left(x_{2}\right) \cdots d \vartheta_{j}\left(x_{j}\right)}{\left(z-x_{1}\right)\left(x_{1}-x_{2}\right) \cdots\left(x_{j-1}-x_{j}\right)\left(z-x_{j}\right)} .
$$

Taking $\left(x_{1}-x_{j}\right)=\left(x_{1}-x_{2}\right)+\cdots+\left(x_{j-1}-x_{j}\right)$, we obtain

$$
\Psi_{n, j}(z)=(-1)^{j-1} \widetilde{\Phi}_{\widetilde{n}, j}(z)+\sum_{k=1}^{j-1}(-1)^{k-1} \widehat{\vartheta}_{j, k}(z) \widetilde{\Phi}_{\widetilde{n}, k}(z), \quad z \in \mathbb{C} \backslash\left(\bigcup_{k=1}^{j} \Delta_{k}\right),
$$

where $\vartheta_{j, k}=\left\langle\vartheta_{j}, \ldots, \vartheta_{k+1}\right\rangle, 1 \leq k \leq j-1$. Substitute $j$ by $i$ in this relation. Using this formula for $i=1, \ldots, j$, we obtain a triangular linear system of equations by which we can express $\widetilde{\Phi}_{\widetilde{n}, j}$ in terms of $\Psi_{n, k}, k=1, \ldots, j$, as indicated in (53).

For the proof of (54), the idea is to untangle the transformations we have introduced in defining the auxiliary Nikishin system. This is done using formulas (12)-(14) backwards. It is sufficient to show that for each $\widetilde{n}$ we have

$$
\widetilde{\Phi}_{\tilde{n}, j}(z)=\Phi_{n, \tau(j)}(z)+\sum_{k=1}^{j-1} \tilde{\ell}_{j, k}(z) \Phi_{n, \tau(k)}(z)+\tilde{\ell}_{j, 0}(z),
$$

where $\tilde{\ell}_{j, k}, k=0, \ldots, j-1$, denote polynomials which do not depend on $n \in \Lambda$ of degree $\leq$ $m-1$. Indeed, from the triangular structure of this relation with respect to $j$, formula (54) follows immediately.

First let us obtain a similar formula for the functions $\widehat{\vartheta}_{1, j}, j=1, \ldots, m$. We will show that for all $j=1,2, \ldots, m$

$$
\widehat{\vartheta}_{1, j}=\widehat{s}_{\tau(j)}+\sum_{k=1}^{j-1} \ell_{j, k} \widehat{s}_{\tau(k)}+\ell_{j, 0},
$$

where $\ell_{j, k}, k=0, \ldots, j-1$, denote polynomials of degree $\leq m-1$ which do not depend on $n \in \Lambda$. The proof of (56) is carried out by induction on the number of measures in the original Nikishin system.

Let us assume that $\Sigma^{0}=\left(\sigma_{1}^{0}\right)$; that is $m=1$. Then for any $n \in \mathbb{Z}_{+}^{1}(*)=\mathbb{Z}_{+}$the associated permutation is $\tau(1)=1$ and $\vartheta_{1}=\sigma_{1}^{0}$. Thus, the formula is trivial. Suppose that the formula is valid for all $j=1,2, \ldots, m-1$ of any Nikishin system of $m-1$ elements $(m \geq 2)$ and $n \in \Lambda \subset \mathbb{Z}_{+}^{m-1}(*)$, where $\Lambda$ is such that all its multi-indices have associated the same permutation. Let us prove that the corresponding statement holds if the original Nikishin system has $m$ elements. 
Let $\Sigma^{0}=\left(\sigma_{1}^{0}, \ldots, \sigma_{m}^{0}\right)$ be an arbitrary Nikishin system of $m \geq 2$ elements and $n^{0} \in \Lambda^{0} \subset \mathbb{Z}_{+}^{m}(*)$. Let $\left(\vartheta_{1}, \ldots, \vartheta_{m}\right)$ be the auxiliary Nikishin system and $\tau^{0}$ the corresponding permutation. For $j=1$

$$
\widehat{\vartheta}_{1,1}(z)=\int_{\Delta_{1}} \frac{d s_{r_{0}}^{0}(x)}{z-x}=\widehat{s}_{\tau(1)}^{0}(z),
$$

and the formula holds true. Let $j=2, \ldots, m$.

Notice that

$$
\widehat{\vartheta}_{1, j}(z)=\int_{\Delta_{1}} \frac{\widehat{\vartheta}_{2, j}(x) d \vartheta_{1}(x)}{z-x}
$$

where $\widehat{\vartheta}_{2, j}$ is the $(j-1)$ th function of the auxiliary Nikishin system $\left(\vartheta_{2}, \ldots, \vartheta_{m}\right)$ associated with the Nikishin system of $m-1$ elements $\Sigma^{1}=\left(\sigma_{2}^{1}, \ldots, \sigma_{m}^{1}\right)$ and the multi-indices $n^{1} \in \Lambda^{1} \subset \mathbb{Z}_{+}^{m-1}(*)$ which are obtained extracting from each $n^{0}$ its $n_{r_{0}}^{0}$ th component (see Section 3). The permutation $\tau^{1}$ of $\{2, \ldots, m\}$ associated with $n^{1} \in \Lambda^{1}$ is

$$
\tau^{1}(j)=\left\{\begin{array}{ccc}
\tau^{0}(j)+1 & \text { if } & \tau^{0}(j)<r_{0} \\
\tau^{0}(j) & \text { if } & \tau^{0}(j)>r_{0}
\end{array}\right.
$$

Using the induction hypothesis it follows that

$$
\widehat{\vartheta}_{2, j}(x)=\sum_{k=2}^{j} \ell_{j, k}(x) \widehat{s}_{\tau^{1}(k)}^{1}(x)+\ell_{j, 1}(x), \quad,
$$

where $\operatorname{deg} \ell_{j, k} \leq m-2, k=1, \ldots, j-1$, and $\ell_{j, j} \equiv 1$. Substituting in (57) we get

$$
\widehat{\vartheta}_{1, j}(z)=\sum_{k=2}^{j} \int_{\Delta_{1}} \frac{\ell_{j, k}(x) \widehat{s}_{\tau^{1}(k)}^{1}(x) d s_{r_{0}}^{0}(x)}{z-x}+\int_{\Delta_{1}} \frac{\ell_{j, 1}(x) d s_{r_{0}}^{0}(x)}{z-x} .
$$

If $r_{0}=1$, then $s_{r_{0}}^{0}=\sigma_{1}^{0}$ and $\Sigma^{1}=\left(\sigma_{2}^{0}, \ldots, \sigma_{m}^{0}\right)$. Consequently, $\tau^{1}(k)=\tau^{0}(k), k=2, \ldots, j, \tau^{0}(1)=$ $1=r_{0}$, and $s_{\tau^{0}(k)}^{1}=s_{2, \tau^{0}(k)}^{0}$. Adding and subtracting in each of the integrals above the polynomials $\ell_{j, k}(z), k=1, \ldots, j$, respectively, it follows that

$$
\begin{gathered}
\widehat{\vartheta}_{1, j}(z)=\sum_{k=2}^{j} \int_{\Delta_{1}} \frac{\left(\ell_{j, k}(x) \mp \ell_{j, k}(z)\right) \widehat{s}_{\tau^{1}(k)}^{1}(x) d s_{r_{0}}^{0}(x)}{z-x}+\int_{\Delta_{1}} \frac{\left(\ell_{j, 1}(x) \mp \ell_{j, 1}(z)\right) d s_{r_{0}}^{0}(x)}{z-x}= \\
\sum_{k=1}^{j} \ell_{j, k}(z) \widehat{s}_{\tau^{0}(k)}^{0}(z)+\ell_{j, 0}(z)
\end{gathered}
$$

which is (56) for this case. In fact, $\ell_{j, j} \equiv 1, \operatorname{deg} \ell_{j, k} \leq m-2, k=2, \ldots, j-1$, and $\operatorname{deg} \ell_{j, 0} \leq m-3$.

Suppose that $r_{0}>1$, then $s_{r_{0}}^{0}=\left\langle\sigma_{1}^{0}, \ldots, \sigma_{r_{0}}^{0}\right\rangle, \Sigma^{1}=\left(\sigma_{2}^{1}, \ldots, \sigma_{m}^{1}\right)$ and $S^{1}=\mathcal{N}\left(\Sigma^{1}\right)=$ $\left(s_{2}^{1}, \ldots, s_{m}^{1}\right)$ (see Section 3). Formula (59) may be rewritten as

$$
\widehat{\vartheta}_{1, j}(z)=\sum_{k=2}^{j} \int_{\Delta_{1}} \frac{\ell_{j, k}(x) \widehat{s}_{\tau^{1}(k)}^{1}(x) \widehat{s}_{2, r_{0}}^{0}(x) d \sigma_{1}^{0}(x)}{z-x}+\int_{\Delta_{1}} \frac{\ell_{j, 0}(x) d s_{r_{0}}^{0}(x)}{z-x} .
$$

Now we need to distinguish those indices $k \in\{2, \ldots, j\}$ such that $\tau^{0}(k)>r_{0}$ from those for which $\tau^{0}(k)<r_{0}$.

Let $k \in\{2, \ldots, j\}$ be such that $\tau^{0}(k)>r_{0}$, then $\tau^{1}(k)=\tau^{0}(k)$ and $s_{\tau^{1}(k)}^{1}=s_{\tau^{0}(k)}^{1}$. Using (14) with $j=0$, it follows that

where $a_{k}$ is a constant, and

$$
\widehat{s}_{\tau^{1}(k)}^{1} \widehat{s}_{2, r_{0}}^{0}=\widehat{s}_{2, \tau^{0}(k)}^{0}-a_{k} \widehat{s}_{2, r_{0}}^{0},
$$

$$
\int_{\Delta_{1}} \frac{\ell_{j, k}(x) \widehat{s}_{\tau^{1}(k)}^{1}(x) \widehat{s}_{2, r_{0}}^{0}(x) d \sigma_{1}^{0}(x)}{z-x}=\ell_{j, k}(z) \widehat{s}_{\tau^{0}(k)}^{0}-a_{k} \ell_{j, k}(z) \widehat{s}_{\tau^{0}(1)}^{0}+\widetilde{\ell}_{j, k}(z),
$$

where $\operatorname{deg} \ell_{j, k} \leq m-2$ and $\operatorname{deg} \widetilde{\ell}_{j, k} \leq m-3$ if $k \in\{2, \ldots, j-1\}$ and if $\tau^{0}(j)>r_{0}$ then $\ell_{j, j} \equiv 1, \widetilde{\ell}_{j, j} \equiv 0$. 
Suppose that $k \in\{2, \ldots, j\}$ is such that $\tau^{0}(k)<r_{0}$, then $\tau^{1}(k)=\tau^{0}(k)+1$ and $s_{\tau^{1}(k)}^{1}=s_{\tau^{0}(k)+1}^{1}$. Using (15) with $j=0$ we have that

$$
\widehat{s}_{\tau^{1}(k)}^{1} \widehat{s}_{2, r_{0}}^{0}=\mathcal{L}_{k} \widehat{s}_{2, r_{0}}^{0}+\sum_{\nu=1}^{\tau^{0}(k)} c_{k, \nu} \widehat{s}_{2, \nu}^{0}, \quad \widehat{s}_{2,1}^{0} \equiv 1
$$

where $c_{k, \nu}$ are constants, $c_{k, \tau^{0}(k)}=1$, and $\mathcal{L}_{k}$ is a first degree polynomial. Notice that if $\tau^{0}(k)<r_{0}$ then for all $\nu=1, \ldots, \tau^{0}(k), \nu=\tau^{0}\left(\nu^{\prime}\right)$ where $\nu^{\prime} \in\{2, \ldots, k\}$, otherwise $n \notin \mathbb{Z}_{+}^{m}(*)$. Proceeding as above we find that

$$
\int_{\Delta_{1}} \frac{\ell_{j, k}(x) \widehat{s}_{\tau^{1}(k)}^{1}(x) \widehat{s}_{2, r_{0}}^{0}(x) d \sigma_{1}^{0}(x)}{z-x}=\left(\ell_{j, k} \mathcal{L}_{k}\right)(z) \widehat{s}_{\tau^{0}(1)}^{0}(z)+\ell_{j, k}(z) \sum_{\nu=1}^{\tau^{0}(k)} c_{k, \nu} \widehat{s}_{\tau^{0}\left(\nu^{\prime}\right)}^{0}(z)+\widetilde{\ell}_{j, k}(z)
$$

where $\operatorname{deg} \ell_{j, k} \mathcal{L}_{k} \leq m-1, \operatorname{deg} \tilde{\ell}_{j, k} \leq m-2$. If $k=j$ and $\tau^{0}(j)<r_{0}$ then $c_{j, \tau^{0}(j)} \ell_{j, j} \equiv 1$. Putting together the relations (60)-(62) and rearranging the terms we obtain (56).

Formula (55) for $j=1$ is trivial since $\widetilde{\Phi}_{\widetilde{n}, 1}=\Phi_{n, \tau(1)}$. For $j=2, \ldots, m$, using (56) we have that

$$
\widetilde{\Phi}_{\tilde{n}, j}(z)=\int_{\Delta_{1}} \frac{\left(\beta_{n} Q_{n}\right)(x)}{\alpha_{n}(x)} \frac{\widehat{\vartheta}_{2, j}(x) d \vartheta_{1}(x)}{z-x}=\int_{\Delta_{1}} \frac{\left(\beta_{n} Q_{n}\right)(x)}{\alpha_{n}(x)} \frac{\left(\sum_{k=2}^{j} \ell_{j, k} \widehat{s}_{\tau^{1}(k)}^{1}+\ell_{j, 0}\right)(x) d \vartheta_{1}(x)}{z-x},
$$

where $\ell_{j, j} \equiv 1$ and $\tau^{1}$ is defined in terms of $\tau=\tau^{0}$ as indicated in (58). To complete the proof of (55) one repeats the arguments used in the last part of the proof of (56) considering separately the cases $j=1$ and $j=2, \ldots, m$. That $\ell_{j, 0} \equiv 0$ if $\min _{1 \leq i \leq m} n_{i} \geq m-1, n \in \Lambda$, is a consequence of orthogonality. With this we conclude the proof.

Take any infinite subsequence $\Lambda^{\prime} \subset \Lambda$ such that the same permutation $\tau$ is associated to all $n \in \Lambda^{\prime}$. Let $\left\{\beta_{n}\right\},\left\{\alpha_{n}\right\}, n \in \Lambda$, satisfy (36). Let $\left(\bar{\mu}_{1}, \ldots, \bar{\mu}_{m}\right)$ be the solution of the equilibrium problem with the initial data (1)-(4) given in Section 5 . For each $j=1, \ldots, m$, set

$$
U_{j}^{\bar{\mu}}=V^{\bar{\mu}_{j}}-V^{\bar{\mu}_{j+1}}-v_{j},
$$

$\left(V^{\widehat{\mu}_{m+1}} \equiv 0\right)$. Notice that in a neighborhood of $z=\infty$ we have

$$
U_{j}^{\bar{\mu}}(z)=\mathcal{O}\left(p_{\tau(j)} \log \frac{1}{|z|}\right) \rightarrow-\infty \text { as } z \rightarrow \infty .
$$

Fix $j \in\{1, \ldots m\}$. For $k=1, \ldots, j$ define the regions

$$
D_{k}^{j}=\left\{z \in D=\overline{\mathbb{C}} \backslash \Delta_{1}: U_{k}^{\bar{\mu}}(z)>U_{i}^{\bar{\mu}}(z), i=1, \ldots, j\right\} .
$$

Some $D_{k}^{j}$ could be empty. By (40) and (53) we have that the following asymptotic formula takes place on $D_{k}^{j}$, except on at most a discrete set of points (where $u_{j, k}=0$ ),

$$
\lim _{n \in \Lambda^{\prime}}\left|\widetilde{\Phi}_{\widetilde{n}, j}\right|^{\frac{1}{n \mid}}=\exp U_{k}^{\bar{\mu}}
$$

Denote

$$
\xi_{j}(z)=\max \left\{U_{k}^{\bar{\mu}}(z): k=1, \ldots j\right\}
$$

then

$$
\lim _{n \in \Lambda^{\prime}}\left|\widetilde{\Phi}_{\tilde{n}, j}(z)\right|^{\frac{1}{|n|}}=\exp \xi_{j}(z), \quad z \in \bigcup_{k=1}^{j} D_{k}^{j},
$$

except on at most a discrete set of points, and

$$
\limsup _{n \in \Lambda^{\prime}}\left|\widetilde{\Phi}_{\widetilde{n}, j}(z)\right|^{\frac{1}{|n|}} \leq \exp \xi_{j}(z), \quad z \in D
$$

Using (51), (52), and (63) it follows that

$$
\lim _{n \in \Lambda^{\prime}}\left|\widetilde{\delta}_{n, j}(z)\right|^{1 /|n|}=\exp \left(V^{\widehat{\mu}_{1}}+f_{1}+\xi_{j}\right)(z), \quad z \in \bigcup_{k=1}^{j} D_{k}^{j} \backslash E,
$$


except on at most a discrete set of points, and

$$
\limsup _{n \in \Lambda^{\prime}}\left|\widetilde{\delta}_{n, j}(z)\right|^{1 /|n|} \leq \exp \left(V^{\widehat{\mu}_{1}}+f_{1}+\xi_{j}\right)(z), \quad z \in D
$$

Formulas (63) and (64) take place uniformly on compact subsets of $D$.

The set

$$
\Omega_{j}^{c}=\left\{z \in D:\left(V^{\widehat{\mu}_{1}}+f_{1}+\xi_{j}\right)(z)<0\right\}
$$

is the domain of convergence to $\widehat{\vartheta}_{1, j}$ of the approximants $\widetilde{R}_{\widetilde{n}, j}$. This set contains a neighborhood of infinity whenever $|\alpha|<1+|\beta|+p_{\tau(j)}$ because

$$
\left(V^{\widehat{\mu}_{1}}+f_{1}+\xi_{j}\right)(z)=\mathcal{O}\left(\left(1+|\beta|+p_{\tau(j)}-|\alpha|\right) \log \frac{1}{|z|}\right) \rightarrow-\infty \quad \text { as } \quad z \rightarrow \infty .
$$

By assumption $|\alpha| \leq 1+|\beta|+p_{\tau(m)}$ since for all $n \in \Lambda, \operatorname{deg} \alpha_{n} \leq|n|+\operatorname{deg} \beta_{n}+\min \left\{n_{i}: i=\right.$ $1, \ldots, m\}$. In order to have convergence on a neighborhood of infinity it is sufficient that $p_{\tau(j)}>$ $p_{\tau(m)}$ or $|\alpha|<1+|\beta|+p_{\tau(m)}$. The convergence is uniform on compact subsets of $\Omega_{j}^{c}$ and the rate is geometric. There can also be a non-empty domain of divergence given by

$$
\Omega_{j}^{d}=\left\{z \in D:\left(V^{\widehat{\mu}_{1}}+f_{1}+\xi_{j}\right)(z)>0\right\} .
$$

THEOREM 7. Let us assume that $\sigma_{1}^{\prime}>0$ almost everywhere on $\Delta_{1}$ and $\sigma_{j} \in \mathbf{R e g}$ for $j=2, \ldots, m$. We also assume that (35) and (36) take place. Take any infinite subsequence $\Lambda^{\prime} \subset \Lambda$ such that the same permutation $\tau$ is associated to all $n \in \Lambda^{\prime}$. Then, for each $j=1, \ldots, m$, we have

$$
\lim _{n \in \Lambda^{\prime}}\left|\Phi_{n, \tau(j)}(z)\right|^{\frac{1}{|n|}}=\exp \xi_{j}(z), \quad z \in \bigcup_{k=1}^{j} D_{k}^{j},
$$

except on at most a discrete set of points, and

$$
\limsup _{n \in \Lambda^{\prime}}\left|\Phi_{n, \tau(j)}(z)\right|^{\frac{1}{|n|}} \leq \exp \xi_{j}(z), \quad z \in D
$$

For the remainders

$$
\lim _{n \in \Lambda^{\prime}}\left|\left(\widehat{s}_{\tau(j)}-R_{n, \tau(j)}\right)(z)\right|^{1 /|n|}=\exp \left(V^{\widehat{\mu}_{1}}+f_{1}+\xi_{j}\right)(z), \quad z \in \bigcup_{k=1}^{j} D_{k}^{j} \backslash E,
$$

except on at most a discrete set of points, and

$$
\limsup _{n \in \Lambda^{\prime}}\left|\left(\widehat{s}_{\tau(j)}-R_{n, \tau(j)}\right)(z)\right|^{1 /|n|} \leq \exp \left(V^{\widehat{\mu}_{1}}+f_{1}+\xi_{j}\right)(z) .
$$

In each case the convergence is uniform on compact subsets of the indicated regions. The regions of convergence and divergence are given by $\Omega_{j}^{c}$ and $\Omega_{j}^{d}$ respectively.

Proof. Formula (65) and the one above it follow from (54) and (63). Notice that (35) implies that $\ell_{j, 0} \equiv 0$ for all $n \in \Lambda$. Formula (66) and the previous one are a direct consequence of (50), (51), and (65).

In order to guarantee a domain of convergence as large as possible, we will introduce an additional restriction on the sequence of polynomials $\left\{\beta_{n}\right\}, n \in \Lambda$, which carry the fixed poles. This is a natural restriction because an arbitrary selection of the fixed poles can spoil convergence.

Let us assume that

$$
\beta \leq\left(\bar{\mu}_{2}+\alpha\right)^{\prime}+\left(2 \theta_{1}+|\beta|-\theta_{2}-|\alpha|\right) \omega_{\Delta_{1}},
$$

where $\left(\bar{\mu}_{2}+\alpha\right)^{\prime}$ denotes the balayage of $\bar{\mu}_{2}+\alpha$ onto $\Delta_{1}$ and $\omega_{\Delta_{1}}$ denotes the equilibrium measure on $\Delta_{1}$. The right hand of $(67)$ is a positive measure since $\theta_{2}+|\alpha| \leq \theta_{2}+1+|\beta|+p_{\tau(m)}=$ $2+|\beta|+p_{\tau(m)}-p_{\tau(1)} \leq 2 \theta_{1}+|\beta|$, because $\theta_{1}=1$ and $p_{\tau(m)}-p_{\tau(1)} \leq 0$. The inequality in (67) means that the measure on the right hand dominates the one on the left on any Borel set. This condition may seem a bit strange since $\bar{\mu}_{2}$, which is on the right hand (67), depends on $\beta$. Nevertheless, the condition may be checked a fortiori once the equilibrium problem is solved. On the other hand, it is sufficient to take

$$
\beta \leq \alpha^{\prime}+\left(2 \theta_{1}+|\beta|-\theta_{2}-|\alpha|\right) \omega_{\Delta_{1}}
$$


in order that (67) takes place. Here, before checking the condition on $\beta$ we only have to determine how much mass we want it to hold and then select any measure with that much mass dominated by the right hand side of the inequality. Certainly, if $\beta=0$ then (67) is satisfied. This is the case when no poles are fixed.

LEMMA 6. Let $\left(\bar{\mu}_{1}, \ldots, \bar{\mu}_{m}\right)$ be the solution of the equilibrium problem with the initial data $(1)-(4)$ given in Section 5. Let us assume that (67) takes place. Then

$$
2 \bar{\mu}_{1}+\beta=\left(\bar{\mu}_{2}+\alpha\right)^{\prime}+\left(2 \theta_{1}+|\beta|-\theta_{2}-|\alpha|\right) \omega_{\Delta_{1}} .
$$

In particular,

$$
\left(W_{1}^{\bar{\mu}}+f_{1}\right)(x)=w_{1}^{\bar{\mu}}, \quad x \in \Delta_{1} .
$$

Proof. According to Theorem 4 we have that

$$
\left(W_{1}^{\bar{\mu}}+f_{1}\right)(x)=\left(2 V^{\bar{\mu}_{1}}+V^{\beta}-V^{\bar{\mu}_{2}+\alpha}\right)(x)=w^{\bar{\mu}_{1}}, \quad x \in \operatorname{supp}\left(\bar{\mu}_{1}\right) .
$$

On the other hand, from Corollary 1 we have that $\lambda=\left(\bar{\mu}_{2}+\alpha\right)^{\prime}+\left(2 \theta_{1}+|\beta|-\theta_{2}-|\alpha|\right) \omega_{\Delta_{1}}$ is the unique measure in $\mathcal{M}_{2 \theta_{1}+|\beta|}\left(\Delta_{1}\right)$ such that

$$
\left(V^{\lambda}-V^{\bar{\mu}_{2}+\alpha}\right)(x)=w^{\lambda}, \quad x \in \operatorname{supp}(\lambda)=\Delta_{1} .
$$

Because of (67) we can write $\lambda=2 \frac{\lambda-\beta}{2}+\beta$ with $\frac{\lambda-\beta}{2} \in \mathcal{M}_{\theta_{1}}\left(\Delta_{1}\right)$ and from the last relation we obtain that

$$
\left(2 V^{(\lambda-\beta) / 2}+V^{\beta}-V^{\bar{\mu}_{2}+\alpha}\right)(x)=w^{\lambda}, \quad x \in \operatorname{supp}(\lambda)=\Delta_{1} .
$$

Using again Corollary 1 with the external field $\left(V^{\beta}-V^{\bar{\mu}_{2}+\alpha}\right)(x)$, from the uniqueness of the solution, we obtain that $\bar{\mu}_{1}=\frac{\lambda-\beta}{2}$ which is the first assertion of this lemma and the second statement immediately follows from the last relation.

Under mild additional restrictions, in the following Corollary we obtain convergence in the largest possible region.

COROllary 2. Let us assume that $\sigma_{1}^{\prime}>0$ almost everywhere on $\Delta_{1}$ and $\sigma_{j} \in \mathbf{R e g}$ for $j=$ $2, \ldots, m$. Let (35), (36) and (67) take place and $|\alpha|<1+|\beta|+p_{\tau(m)}$. Take any infinite subsequence $\Lambda^{\prime} \subset \Lambda$ such that the same permutation $\tau$ is associated to all $n \in \Lambda^{\prime}$. Then, $\Omega_{j}^{c}=D$ for each $j=1, \ldots, m$. For each $j$ the number of non-empty regions $D_{k}^{j}, k=1, \ldots, j$, in which we have different asymptotic formulas for $\left\{\left|\Phi_{n, \tau(j)}\right|^{1 /|n|}\right\},\left\{\left|\delta_{n, \tau(j)}\right|^{1 /|n|}\right\}, n \in \Lambda^{\prime}$, as described in Theorem 7 is at most one more than the number of strict inequalities in the sequence $p_{\tau(1)} \geq \cdots \geq p_{\tau(j)}$.

Proof. For $j=1$ we have that (see (66))

$$
\limsup _{n \in \Lambda^{\prime}}\left|\delta_{n, \tau(1)}(z)\right|^{1 /|n|} \leq \exp \left(W_{1}^{\widehat{\mu}}(z)-w_{1}^{\widehat{\mu}}\right), \quad z \in D .
$$

From Lemma 6 we have that $W_{1}^{\widehat{\mu}}-w_{1}^{\widehat{\mu}}=0$ on $\Delta_{1}$. On the other hand, $W_{1}^{\widehat{\mu}}$ is subharmonic in $\overline{\mathbb{C}} \backslash \Delta_{1}$ and $W_{1}^{\widehat{\mu}}(z)=O\left(\left(1+|\beta|+p_{\tau(1)}-|\alpha|\right) \log \frac{1}{|z|}\right) \rightarrow-\infty$ as $z \rightarrow \infty$. Therefore, $W_{1}^{\widehat{\mu}}-w_{1}^{\widehat{\mu}}<0$ on $\overline{\mathbb{C}} \backslash \Delta_{1}$ which is what we needed to prove.

Let us assume that the Corollary is valid for $j-1$ where $j \in\{2, \ldots, m\}$ and let us prove that it also holds for $j$. From Theorem 7 we know that

$$
\limsup _{n \in \Lambda^{\prime}}\left|\delta_{n, \tau(j)}(z)\right|^{1 /|n|} \leq \exp \left(V_{1}^{\widehat{\mu}}+f_{1}+\xi_{j}\right)(z), \quad z \in D .
$$

Obviously, $\xi_{j}(z)=\max \left\{\xi_{j-1}(z), U_{j}(z)\right\}$. Consider the difference $U_{j}(z)-U_{j-1}(z)=W_{j}^{\bar{\mu}}(z)-w_{j}^{\bar{\mu}}=$ $O\left(\left(p_{\tau(j)}-p_{\tau(j-1)}\right) \log \frac{1}{|z|}\right), z \rightarrow \infty$.

If $p_{\tau(j)}=p_{\tau(j-1)}$ then $W_{j}^{\bar{\mu}}(z)-w_{j}^{\bar{\mu}}$ is subharmonic in $\overline{\mathbb{C}} \backslash \operatorname{supp}\left(\bar{\mu}_{j}\right)$ and equals zero on $\operatorname{supp}\left(\bar{\mu}_{j}\right)$. Hence, $U_{j}(z) \leq U_{j-1}(z) \leq \xi_{j-1}(z)$ on $\overline{\mathbb{C}} \backslash \operatorname{supp}\left(\bar{\mu}_{j}\right)$. Therefore, using the equilibrium condition, $U_{j}(z)=U_{j-1}(z)$ on $\Delta_{j}$ and $U_{j}(z)<U_{j-1}(z)$ on $\overline{\mathbb{C}} \backslash \Delta_{j}$. In this case, $\xi_{j}(z)=\xi_{j-1}(z), z \in D$, and the conclusion follows from the induction hypothesis.

If $p_{\tau(j)}<p_{\tau(j-1)}$, in a neighborhood of $\infty$ we have $U_{j}(z)>U_{j-1}(z)$ since $\left(p_{\tau(j)}-p_{\tau(j-1)}\right) \log \frac{1}{|z|} \rightarrow$ $+\infty$ as $z \rightarrow \infty$. Let $\Gamma=\left\{z \in D: U_{j}(z)=U_{j-1}(z)\right\}$. This set contains $\operatorname{supp}\left(\bar{\mu}_{j}\right)$ and divides $D$ in two domains $\Omega_{1}=\left\{z \in D: U_{j}(z)>U_{j-1}(z)\right\}$, which contains $z=\infty$, and 
$\Omega_{2}=\left\{z \in D: U_{j}(z)<U_{j-1}(z)\right\}$. Since $U_{j-1}(z) \leq \xi_{j-1}(z)$, on $\Omega_{2} \cup \Gamma$ we have that $\xi_{j-1}(z)=\xi_{j}(z)$ and thus $\left(V^{\bar{\mu}_{1}}+f_{1}+\xi_{j}\right)<0$. On $\Omega_{1}$ the function $V^{\bar{\mu}_{1}}+f_{1}+U_{j}$ is subharmonic and on its boundary $\Gamma$ equals $V^{\bar{\mu}_{1}}+f_{1}+U_{j-1}<0$. Since $\left(V^{\bar{\mu}_{1}}+f_{1}+U_{j}\right)(z) \rightarrow-\infty$ as $z \rightarrow \infty$ it follows that on $\Omega_{1}$ we have $\left(V^{\bar{\mu}_{1}}+f_{1}+U_{j}\right)(z)<0$. Therefore, $\left(V^{\bar{\mu}_{1}}+f_{1}+\xi_{j}\right)<0$ on $\Omega_{1}$. With this we conclude the proof.

\section{REFERENCES}

[1] A. Branquinho, J. Bustamante, A. Folquié, and G. López, Normal indices in Nikishin systems, J. of Approx. Theory, 124 (2003), 254-263.

[2] ZH. Bustamante And G. López Lagomasino, Hermite-Padé approximation for Nikishin systems of analytic functions, Russian Acad. Sci. Sb. Math. 77 (1994), 367-384.

[3] K. Driver And H. Stahl, Simultaneous rational approximants to Nikishin systems II, Acta Sci. Math. 61 (1995), 261-284.

[4] U. Fidalgo and G. López Lagomasino, On perfect Nikishin systems, Comp. Meth. in Function Theory 2 (2002), 415-426.

[5] U. Fidalgo, J. Illán, And G. López Lagomasino, Hermite-Padé approximants and simultaneous quadrature formulas, J. of Approx. Theory, (2004), .

[6] A. A. Gonchar And E. A. Rakhmanov, On equilibrium problems for vector potentials, Russian Math. Surveys 40 (1985), 183-184.

[7] A. A. Gonchar And E. A. Rakhmanov, Equilibrium measure and the distribution of zeros of extremal polynomials, Math. USSR Sb. 53 (1986), 119-130.

[8] A.A. Gonchar, E.A. Rakhmanov, and V.N. Sorokin, Hermite-Padé Approximants for systems of Markovtype functions, Sbornik Mathematics 188 (1997), 33-58.

[9] M. G. Krein And A. A. Nudel'man, "The Markov Moment problem and extremal problems", Translations of Math. Monographs, Vol. 50, Amer. Math. Soc., Providence, R. I., 1977.

[10] N. S. LAndKof, "Foundations of Modern Potential Theory", Grundlehren der Mathenatischen Wissenschaften, Vol. 190, Springer Verlag, New York, 1972.

[11] E. M. Nikishin, On simultaneous Padé Approximants, Math. USSR Sb. Vol. 41 (1982), $409-425$.

[12] E.M. Nikishin And V.N. Sorokin, "Rational Approximations and Orthogonality". Translations of Math. Monographs, Vol. 92, Amer. Math. Soc., Providence, R. I., 1991.

[13] H. Stahl and V. Totik, “General Orthogonal Polynomials,” Cambridge University Press, Cambridge, 1992.

(Ulises) Departamento de Matemáticas, UniversidadCarlos III de Madrid, C/ Universidad 30,28911 LEGANÉs, SPAIN.

E-mail address, Ulises: ulises@math.uc3m.es

(Guillermo) Departamento de Matemáticas, Universidad Carlos III, C/ Universidad 30, 28911 Leganés, SPAIN.

E-mail address, Guillermo: lago@math.uc3m.es 